\title{
Las prestaciones económicas de dependencia en Gipuzkoa: concurrencia, impacto y supervisión
}

\section{Siadeco Ikerketa Aplikatua}

<siadeco@siadeco.net>

Artikulu hau 2012an Gipuzkoako Foru Aldundiaren Gizarte Politikako Departamentuarentzat egindako ikerketa batean oinarritzen da. Bertan mendekotasunaren balorazioetan zerikusia duten prestazio ekonomikoen eraginaren azterketa kualitatiboa egin da. Lehenengo, azterketa objektua aurkezten da, Gipuzkoan emandako prestazio ekonomikoak eta horietan inbertitutako baliabideak kuantifikatuz. Horrez gain, prestazio horien onuradunek osatzen duten kolektiboaren ezaugarri nagusiak aztertzen dira. Ondoren, honakoak aztertzen dira: prestazio horiek bestelako programa eta gizarte-zerbitzuekin uztartzeko modua, onuradunengan nahiz beren familiengan duten eragina, eta zaintzaren kalitate eta autonomiaren sustapenaren inguruko ikuskapena. Amaitzeko, aztertu diren alderdiak hobetu eta optimizatzeko proposamena egiten da.

\section{HITZ-GAKOAK:}

Mendekotasuna, ikerketa kualitatiboa, prestazio ekonomikoak, gizarte-zerbitzuak, eragina, Gipuzkoa.
Este artículo se basa en un estudio realizado en 2012 para el Departamento de Política Social de la Diputación Foral de Gipuzkoa, centrado en el análisis cualitativo del impacto de las prestaciones económicas asociadas a las valoraciones de dependencia. En primer lugar, se presenta el objeto de estudio, cuantificando las prestaciones económicas otorgadas en Gipuzkoa y los recursos invertidos en ellas, y proporcionando una caracterización básica del colectivo de personas beneficiarias de esas ayudas. Después, se examina la forma en la que tales prestaciones concurren con otros programas o servicios sociales, el efecto que tienen sobre las personas beneficiarias y sus familias, y la supervisión de la calidad de la atención y promoción de la autonomía. Para terminar, se plantea una propuesta de mejora u optimización respecto a las cuestiones analizadas.

\section{Palabras Clave:}

Dependencia, estudio cualitativo, prestaciones económicas, servicios sociales, impacto, Gipuzkoa. 


\section{Planteamiento y contextualización}

\subsection{Planteamiento}

El sustrato básico de este artículo es el estudio ${ }^{1}$ realizado en 2012 por Siadeco para el Departamento de Política Social de la Diputación Foral de Gipuzkoa, centrado en el análisis cualitativo del impacto de las prestaciones económicas asociadas a las valoraciones de dependencia implementadas a partir de la Ley 39/2006 de Promoción de la Autonomía Personal y Atención a las personas en situación de dependencia (en adelante, LAPAD) ${ }^{2}$.

Para la realización de dicho estudio, Siadeco, además de recopilar y analizar información documental, alimentó informativamente una estructura metodológica cualitativa basada en la realización de entrevistas estructuradas en profundidad con muestras estratégicas ${ }^{3}$ de informantes, obtenidas básicamente a partir de los siguientes colectivos:

- Profesionales de los servicios sociales implicados (técnicos forales y municipales, y técnicos de diversas entidades sociales 0 asociaciones relacionadas con colectivos de personas dependientes), a los que a lo largo del artículo denominaremos 'agentes de intervención'. En total, se realizaron 29 entrevistas en este colectivo.

- Familiares y personas cuidadoras de personas dependientes perceptoras de prestaciones económicas para cuidados en el entorno familiar y apoyo a cuidadores no profesionales (PECE) y prestaciones económicas de asistencia personal (PEAP) en Gipuzkoa. En total, se entrevistó a 60 familias perceptoras de la PECE y a 10 perceptoras de la PEAP.

A partir de la información directa cualitativa obtenida, se llevaron a cabo diversos tipos de análisis e interpretaciones, y en este artículo se tratan de exponer, de forma sintética y divulgativa, algunos de los aspectos que consideramos centrales en dicho estudio. Así, después de conceptualizar y dimensionar cuantitativamente el objeto de análisis, aportando datos sobre

${ }^{1}$ Siadeco, Estudio cualitativo sobre el impacto de las prestaciones económicas de dependencia en Gipuzkoa, Donostia-San Sebastián, Diputación Foral de Gipuzkoa, 2012.

${ }^{2}$ La Ley 39/2006 de Promoción de la Autonomía Personal y Atención a las personas en situación de dependencia se dirige a las personas que se encuentran en un estado de dependencia por razones ligadas a la falta o pérdida de autonomía física, sensorial, psíquica o intelectual y necesitan asistencia o ayudas importantes a fin de realizar las actividades ordinarias de la vida diaria y, de modo particular, las referentes al cuidado personal. Ésta es la ley social más reciente de carácter estatal que se aplica en el ámbito de las personas con discapacidad y que intenta contribuir a configurar un Sistema para la Autonomía y Atención a la Dependencia (SAAD) como el cuarto pilar del Estado de bienestar (tras el sistema de salud, el sistema educativo y el sistema de pensiones, desarrollados en la década de los ochenta), dando amparo a un nuevo derecho subjetivo de ciudadanía, a saber: el derecho a la promoción de la autonomía personal y a la atención a las personas en situación de dependencia por parte del Estado.

${ }^{3}$ Hacemos referencia a muestras estratégicas, y no a muestras estadísticamente representativas, por tratarse de un estudio cualitativo, y no de un estudio cuantitativo. las prestaciones económicas otorgadas en Gipuzkoa hasta aquel momento y los recursos invertidos en ellas, y proporcionando una caracterización básica del colectivo de personas beneficiarias, pasamos a analizar la forma en la que tales prestaciones económicas concurren con otros programas o servicios sociales, el efecto que tienen sobre las personas beneficiarias y sus familias, y la supervisión de la calidad de la atención y la promoción de la autonomía. Para terminar, se plantea una propuesta de mejora u optimización respecto a las cuestiones analizadas.

\subsection{Tipos de prestaciones de dependencia}

Cabe recordar que las prestaciones económicas de dependencia a las que estamos haciendo referencia son de tres tipos 4 :

- Prestación económica vinculada a la adquisición de un servicio (PEVS): cuando la persona dependiente lleva tres meses en lista de espera para acceder a un servicio de centro de día o una residencia permanente, y no se dispone de plaza, percibirá esta prestación para contribuir a la cobertura del coste de la plaza privada que se haya visto obligada a contratar.

- Prestación económica para cuidados en el entorno familiar y apoyo a cuidadores no profesionales (PECE): está destinada a contribuir a la cobertura de los gastos derivados de la atención a la persona dependiente por parte de un cuidador de su entorno familiar.

- Prestación económica de asistencia personal (PEAP): está dirigida a contribuir a la cobertura de los gastos derivados de la contratación de un/a asistente personal que facilite el acceso a la educación o al trabajo, y posibilite una mayor autonomía en el ejercicio de las actividades básicas de la vida diaria 5 .

A partir del Baremo de Valoración de la Dependencia (BVD), es necesario acreditar cierto grado y nivel de dependencia para acceder al Sistema para la Autonomía y Atención a la Dependencia (SAAD). El BVD posibilita la valoración funcional de actividades básicas de la vida diaria (ABVD) y de algunas actividades instrumentales (AIVD) no avanzadas. Pero desde nuestro punto de vista, es un instrumento escorado a la valoración de la situación de la movilidad física de la persona, que condiciona la autonomía personal en ABVD como lavarse, alimentarse o vestirse.

4 Las finalidades y requisitos de los distintos tipos de prestaciones fueron reguladas por la Diputación Foral de Gipuzkoa mediante el Decreto Foral 25/2009, de 21 de julio.

${ }_{5}^{5}$ Hay que tener en cuenta que, en el momento realizar el estudio, para poder acceder a este tipo de prestación, la persona dependiente debía contar con una valoración de dependencia de grado III. Pero a partir de las medidas de racionalización del sistema de dependencia contempladas en el Real Decreto Ley $20 / 2012$, se modifica el artículo 19 de la LAPAD, pues se establece que la prestación económica de asistencia personal (PEAP) tiene como finalidad la promoción de la autonomía de las personas en situación de dependencia en cualquiera de sus grados. 


\begin{tabular}{|c|c|c|c|}
\hline \multicolumn{4}{|c|}{$\begin{array}{l}\text { Cuadro 1. Caracterización de grados y niveles }{ }^{6} \text { de } \\
\text { dependencia y puntuación BVD asociada }\end{array}$} \\
\hline Grados & Necesidades & Niveles & $\begin{array}{l}\text { Puntuación } \\
\text { BVD }\end{array}$ \\
\hline \multirow{2}{*}{$\begin{array}{l}\text { Grado I: } \\
\text { dependencia } \\
\text { moderada }\end{array}$} & \multirow{2}{*}{$\begin{array}{l}\text { Necesita ayuda } \\
\text { para realizar alguna } \\
\text { actividad básica } \\
\text { de la vida diaria } \\
\text { al menos una vez } \\
\text { al día, o tiene } \\
\text { necesidades de } \\
\text { apoyo intermitente } \\
\text { o limitado para } \\
\text { su autonomía } \\
\text { personal. }\end{array}$} & Nivel 1 & $25-39$ \\
\hline & & Nivel 2 & $40-49$ \\
\hline \multirow{2}{*}{$\begin{array}{l}\text { Grado II: } \\
\text { dependencia } \\
\text { severa }\end{array}$} & \multirow{2}{*}{$\begin{array}{l}\text { Necesita ayuda } \\
\text { para realizar varias } \\
\text { actividades básicas } \\
\text { de la vida diaria dos } \\
\text { o tres veces al día, } \\
\text { pero no requiere el } \\
\text { apoyo permanente } \\
\text { de un cuidador, o } \\
\text { tiene necesidades } \\
\text { de apoyo extenso } \\
\text { para su autonomía } \\
\text { personal. }\end{array}$} & Nivel 1 & $50-64$ \\
\hline & & Nivel 2 & $65-74$ \\
\hline \multirow{2}{*}{$\begin{array}{l}\text { Grado III: gran } \\
\text { dependencia }\end{array}$} & \multirow{2}{*}{$\begin{array}{l}\text { Necesita ayuda } \\
\text { para realizar } \\
\text { varias actividades } \\
\text { básicas de la vida } \\
\text { diaria varias veces } \\
\text { al día y, por su } \\
\text { pérdida total de } \\
\text { autonomía física, } \\
\text { mental, intelectual } \\
\text { o sensorial, } \\
\text { necesita el apoyo } \\
\text { indispensable y } \\
\text { continuo de otra } \\
\text { persona o tiene } \\
\text { necesidades de } \\
\text { apoyo generalizado } \\
\text { para su autonomía } \\
\text { personal. }\end{array}$} & Nivel 1 & $75-89$ \\
\hline & & Nivel 2 & $90-100$ \\
\hline
\end{tabular}

Fuente: Elaboración propia a partir del Real Decreto 504/2007, de 20 de abril, por el que se aprueba el Baremo de Valoración de la Situación de Dependencia.
En Gipuzkoa, las cuantías máximas de las prestaciones económicas de dependencia se determinan anualmente, mediante acuerdo del Consejo de Diputados. En la Tabla 1, se refleja la evolución de tales cuantías económicas máximas, en función del grado y nivel de dependencia, y su calendario de implantación en Gipuzkoa. El importe de la prestación económica de dependencia a la que se puede tener acceso se determinará también en función de los rendimientos declarados en el impuesto sobre la renta de las personas físicas, pudiendo, en su caso, reducirse un $10 \%, 20 \% 030 \%$, tal como se explica en la Tabla 2.

Tabla 2. Cuantía máxima de percepción de las prestaciones económicas de dependencia, según los rendimientos de la persona beneficiaria, y evolución de las cantidades de referencia para la aplicación de las reducciones. Gipuzkoa, 2008-2011 (\%)

\begin{tabular}{|c|c|c|c|c|c|}
\hline \multirow{2}{*}{$\begin{array}{l}\text { Rendimientos } \\
\text { de la persona } \\
\text { beneficiaria }\end{array}$} & \multirow{2}{*}{$\begin{array}{c}\text { Cuantía } \\
\text { máxima } \\
(\%)\end{array}$} & \multicolumn{4}{|c|}{$\begin{array}{l}\text { Cantidades de referencia para la } \\
\text { aplicación de las reducciones }(€)\end{array}$} \\
\hline & & 2008 & 2009 & 2010 & 2011 \\
\hline $\begin{array}{l}>400,0 \% \text { del } \\
\text { SMI }\end{array}$ & 70 & 133.600 & $>34.944$ & 335.465 & $>35.918$ \\
\hline $\begin{array}{l}300,01-400,0 \% \\
\text { del SMI }\end{array}$ & 80 & $\begin{array}{l}25.200- \\
33.600\end{array}$ & $\begin{array}{c}26.208- \\
34.944\end{array}$ & $\begin{array}{l}26.599- \\
35.465\end{array}$ & $\begin{array}{c}26.939- \\
35.918\end{array}$ \\
\hline $\begin{array}{l}250,01-300,0 \% \\
\text { del SMI }\end{array}$ & 90 & $\begin{array}{l}21.000- \\
25.200\end{array}$ & $\begin{array}{l}21.840- \\
26.208\end{array}$ & $\begin{array}{l}22.165^{-} \\
26.599\end{array}$ & $\begin{array}{l}22.449- \\
26.939\end{array}$ \\
\hline $\begin{array}{l}\leq 250,0 \% \text { del } \\
\text { SMI }\end{array}$ & 100 & $<21.000$ & $<21.840$ & $<22.166$ & $<22,449$ \\
\hline \multirow{3}{*}{$\begin{array}{l}\text { Salario mínimo } \\
\text { interprofesional } \\
\text { (SMI) }\end{array}$} & Anual & $8.400,00$ & $8.736,00$ & $8.866,20$ & $8.979,60$ \\
\hline & Mensual & 600,00 & 624,00 & 633,30 & 641,40 \\
\hline & Diario & 20,00 & 20,80 & 21,11 & 21,38 \\
\hline
\end{tabular}

Fuente: Elaboración propia a partir de datos de la Diputación Foral de Gipuzkoa.

Tabla 1. Evolución del importe mensual máximo de las prestaciones económicas de dependencia, según tipo de prestación y año de valoración. Gipuzkoa, 2007-2011 (euros)

\begin{tabular}{|c|c|c|c|c|c|c|c|c|c|c|c|c|c|c|c|}
\hline \multirow{2}{*}{\multicolumn{3}{|c|}{$\begin{array}{l}\text { Valoraciones de } \\
\text { dependencia (BVD): } \\
\text { grados y niveles }\end{array}$}} & \multirow{3}{*}{ 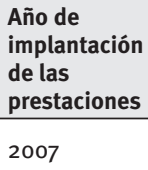 } & \multicolumn{4}{|c|}{$\begin{array}{c}\text { Para cuidados en el entorno } \\
\text { familiar (PECE) }\end{array}$} & \multicolumn{4}{|c|}{ De asistencia personal (PEAP) } & \multicolumn{4}{|c|}{$\begin{array}{c}\text { Vinculadas a la adquisición de } \\
\text { un servicio (PEVS) }\end{array}$} \\
\hline & & & & 2007 & 2008 & 2009 & 2011 & 2007 & 2008 & 2009 & 2011 & 2007 & 2008 & 2009 & 2011 \\
\hline 1 & \multirow{2}{*}{ Grado III } & nivel 12 & & 487,00 & 506,96 & 519,13 & 520,69 & 780,00 & 811,98 & 831,47 & 833,96 & 780,00 & 811,98 & 831,47 & 833,96 \\
\hline 2 & & nivel 11 & 2007 & 390,00 & 405,99 & 415,73 & 416,98 & 585,00 & 608,98 & 623,60 & 625,47 & 585,00 & 608,98 & 623,60 & 625,47 \\
\hline 3 & \multirow{2}{*}{ Grado II } & nivel 12 & 2008 & - & 328,36 & 336,24 & 337,25 & - & - & - & - & - & 450,00 & 460,80 & 462,18 \\
\hline 4 & & nivel 11 & 2009 & - & - & 300,00 & 300,90 & - & - & - & - & - & - & 400,00 & 401,20 \\
\hline 5 & Grado I & nivel 12 & 2011 & - & - & - & 180,00 & - & - & - & - & - & - & - & 300,00 \\
\hline
\end{tabular}

Fuente: Elaboración propia a partir de datos del Departamento de Política Social de la Diputación Foral de Gipuzkoa. 
De la Tabla 2 se desprende que el nivel de ingresos de la persona dependiente incide 'escasamente' en el acceso a las prestaciones económicas de dependencia. De este modo, la situación de necesidad económica no es un criterio determinante para el acceso a la prestación. Este planteamiento 'choca' con formulaciones e intervenciones anteriores de los servicios sociales, que primaban la concesión de ayuda económica asociada a situaciones de necesidad o precariedad. De hecho, entre los agentes que intervienen sobre la población dependiente se constató un discurso que señalaba la necesidad de un desarrollo normativo que estableciera una limitación al acceso a las prestaciones económicas en función de la situación económica y patrimonial de las personas dependientes y su entorno familiar, basándose en el argumento de la eficiencia en el uso de los recursos públicos, sobre la base del planteamiento de que no todas las personas dependientes tienen la misma necesidad de acceder a las prestaciones económicas: la funcionalidad de estas prestaciones difiere significativamente según la situación económica de las personas dependientes y su entorno familiar.

\subsection{La contextualización de las prestaciones económicas de dependencia en los servicios sociales de Gipuzkoa}

La incorporación de las prestaciones económicas a las medidas de apoyo del Sistema para la Autonomía y Atención a la Dependencia (SAAD) parece obedecer a una tendencia generalizada en los modelos occidentales de atención a la dependencia, referidos más comúnmente en el ámbito internacional como modelos de cuidados de larga duración 7 . En nuestro ámbito, no eran una novedad, pero sí lo era el hecho de que se integraran en un sistema específico de atención a este tipo de necesidades y, sobre todo, que se articularan, como el resto de los apoyos previstos, como un derecho. Por lo demás, tenían precedentes directos en todo el Estado. En Gipuzkoa, de hecho, ya existían prestaciones económicas orientadas a las mismas finalidades:

- La prestación económica de cuidados en el entorno (PECE) tiene su precedente en la prestación económica que se concedía, desde 1996, en el marco del programa Sendian de apoyo a familiares cuidadores de dependientes, y a la que sustituye desde su entrada en vigor, si bien la segunda sigue prestándose a las personas que en su día accedieron a ella.

- La prestación económica de asistencia personal (PEAP) tiene un precedente directo en la prestación económica asociada al Programa de Vida Independiente, iniciado en 2004 y que se diseñó para facilitar a algunas personas con discapacidad la salida de un centro residencial o la emancipación del domicilio familiar (gastos de alojamiento, desplazamientos, asistentes). Actualmente, este programa sigue en vigor.

7 Una amplia información sobre este Sistema puede obtenerse en el Portal de la Dependencia del Imserso (〈http://www.dependencia.imserso.es/dependencia_01/ >)
- La prestación económica vinculada a un servicio (PEVS) tiene su precedente en una ayuda económica que, ante la insuficiencia de la oferta pública residencial, se puso en marcha en 2005 en dos comarcas del Territorio Histórico (Bidasoaldea y Oarsoaldea). Esa ayuda, que otorgaba la propia Diputación Foral de Gipuzkoa, actualmente no está en vigor.

\section{Prestaciones económicas otorgadas, recursos invertidos y caracterización de las personas beneficiarias}

\subsection{Prestaciones económicas otorgadas}

En septiembre de 2011, un total de 11.464 personas eran beneficiarias de algún tipo de prestación económica de dependencia en Gipuzkoa; de ellas, el 89,4\%, (10.251 personas), recibían prestaciones para cuidados en el entorno familiar (PECE), el 9,4\% (1.075 personas) prestaciones de asistencia personal (PEAP), y el 1,2\% (138 personas) prestaciones vinculadas a la adquisición de un servicio (PEVS). El conjunto de personas beneficiarias de alguna de estas prestaciones económicas representaba una tasa de 162,1 personas por cada 10.000 habitantes.

Entre diciembre de 2008 y septiembre de 2011, se registró un incremento del 152,9\% en el número de personas beneficiarias de prestaciones económicas de Gipuzkoa, si bien el mayor aumento, correspondió al período 2008-2009 (crecimiento del 87,6\%).

Las personas beneficiarias de la PECE representaban la gran mayoría del colectivo total de personas perceptoras de estas prestaciones (entre el $85 \%$ y el $89 \%$ ), y se observa, además, que año tras año su peso absoluto y relativo era cada vez mayor.

Respecto a la valoración del grado de dependencia, el 42,6\% (4.884 personas) estaban valoradas con el grado III o gran dependencia; el 46,4\% (5.322 personas), con el grado II o dependencia severa; y el $11 \%$ (1.258 personas), con el grado I o dependencia moderada. Teniendo en cuenta tanto el grado de dependencia como el tipo de prestación económica que percibían, se observa (Gráfico 1) que el 45,9\% del total de las personas beneficiarias eran dependientes de grado II que percibían la prestación económica de cuidado en el entorno (PECE); y otro 32,6\%, personas dependientes valoradas con el grado III que también recibían la $\mathrm{PECE}$.

De las personas perceptoras de prestaciones económicas reconocidas con grado III de dependencia, el $76,5 \%$ recibían la PECE; el $22 \%$, la PEAP; y el $1,4 \%$, la PEVS. De las personas perceptoras reconocidas con un grado I o grado II de dependencia, prácticamente la totalidad (99\% o más) recibían la PECE $^{8}$ (Gráfico 2).

${ }^{8}$ Nótese que en 2011, año al que se refieren estos datos, la PEAP sólo era accesible a dependientes de grado III, mientras que las personas que demandaban la PEVS eran un colectivo muy minoritario. 
Tabla 3. Evolución de las personas beneficiaras de prestaciones económicas de dependencia, por tipo de prestación. Gipuzkoa, 2008-2011*

\begin{tabular}{|c|c|c|c|c|c|c|c|c|c|c|c|c|}
\hline \multirow{3}{*}{$\begin{array}{l}\text { Tipos de prestaciones } \\
\text { económicas }\end{array}$} & \multicolumn{11}{|c|}{ Beneficiarios del último mes } & \multirow{3}{*}{$\Delta(\%)$} \\
\hline & \multicolumn{2}{|c|}{2008} & \multicolumn{3}{|c|}{2009} & \multicolumn{3}{|c|}{2010} & \multicolumn{3}{|c|}{2011} & \\
\hline & Abs. & $\%$ & Abs. & $\%$ & $\Delta(\%)$ & Abs. & $\%$ & $\Delta(\%)$ & Abs. & $\%$ & $\Delta(\%)$ & \\
\hline PECE & 3.853 & 85,0 & 7.529 & 88,5 & 95,4 & 8.066 & 88,2 & 7,1 & 10.251 & 89,4 & 27,1 & 166,05 \\
\hline PEAP & 582 & 12,8 & 836 & 9,8 & 43,6 & 960 & 10,5 & 14,8 & 1.075 & 9,4 & 12,0 & 84,71 \\
\hline PEVS & 98 & 2,2 & 138 & 1,6 & 40,8 & 121 & 1,3 & $-12,3$ & 138 & 1,2 & 14,1 & 40,82 \\
\hline Total & 4.533 & 100,0 & 8.503 & 100,0 & 87,6 & 9.147 & 100,0 & 7,6 & 11.464 & 100,0 & 25,3 & 152,90 \\
\hline
\end{tabular}

PECE: prestación para cuidados en el entorno familiar. PEAP: prestación de asistencia personal. PEVS: prestación vinculada a un servicio. * Los datos de 2008 y 2009 son a 31 de diciembre; los de 2010, a 30 de junio de ese año; y los de 2011, a septiembre de ese año. Fuente: Elaboración propia a partir de datos del Departamento de Política Social de la Diputación Foral de Gipuzkoa.

Gráfico 1. Distribución de las prestaciones económicas, por tipo de prestación y grados de dependencia de las personas beneficiarias. Gipuzkoa, septiembre de 2011

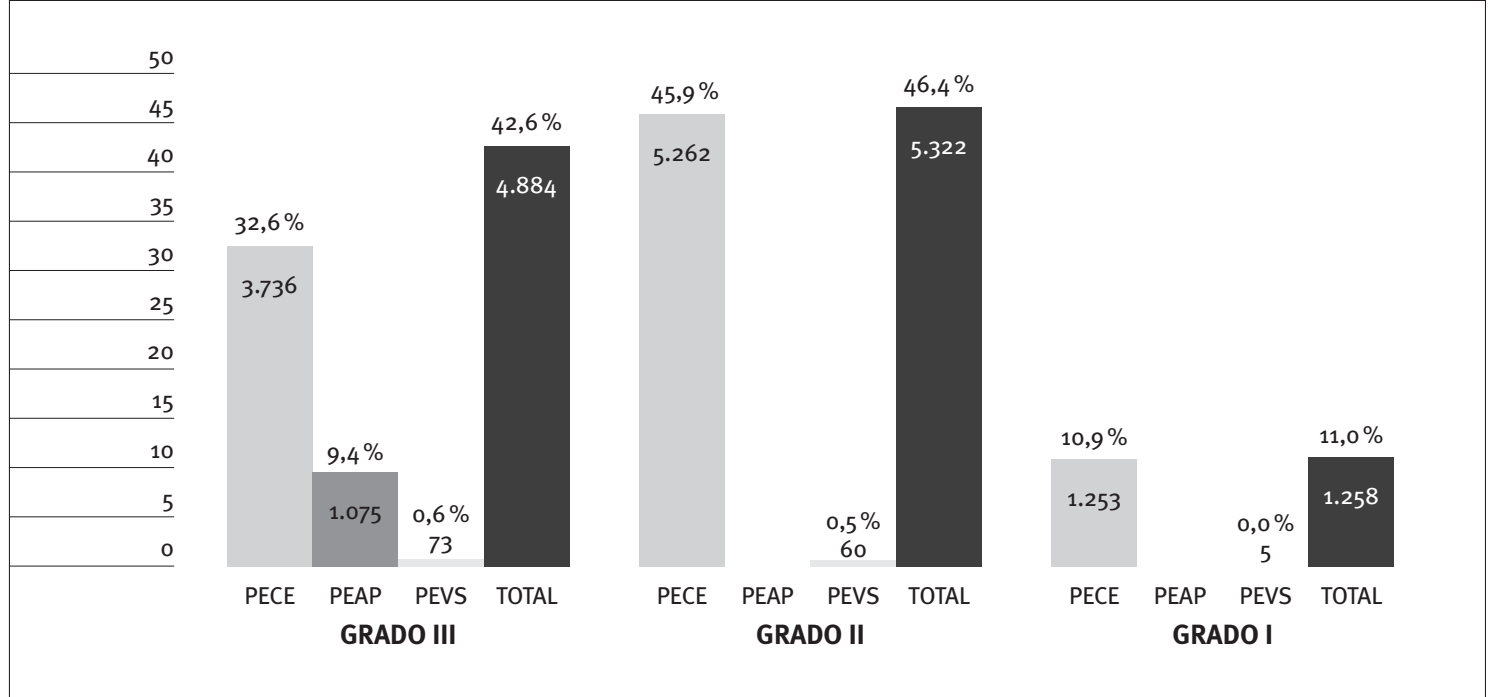

PECE: prestación para cuidados en el entorno familiar. PEAP: prestación de asistencia personal. PEVS: prestación vinculada a un servicio. Fuente: Elaboración propia a partir de datos del Departamento de Política Social de la Diputación Foral de Gipuzkoa.

Gráfico 2. Distribución de las prestaciones económicas, por grados de dependencia de las personas beneficiarias. Gipuzkoa, septiembre de 2011

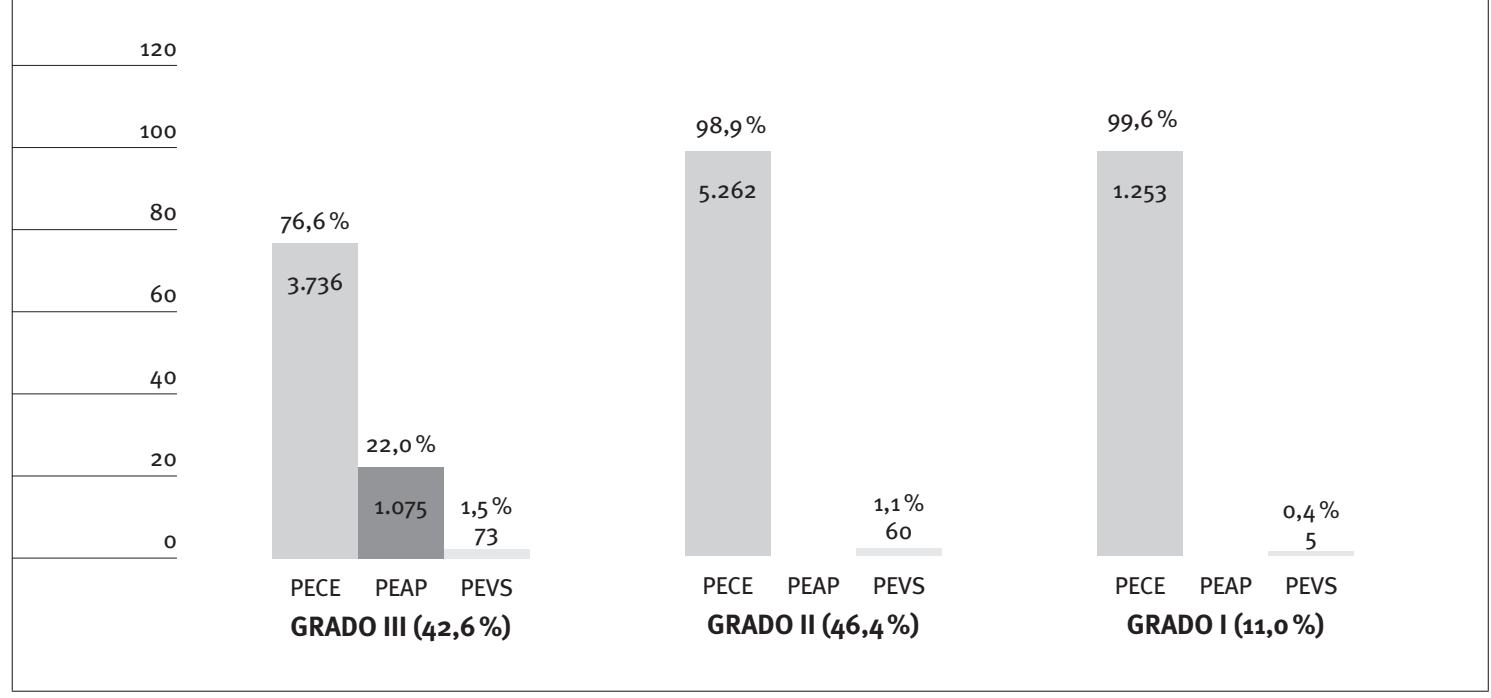

PECE: prestación para cuidados en el entorno familiar. PEAP: prestación de asistencia personal. PEVS: prestación vinculada a un servicio. Fuente: Elaboración propia a partir de datos del Departamento de Política Social de la Diputación Foral de Gipuzkoa. 

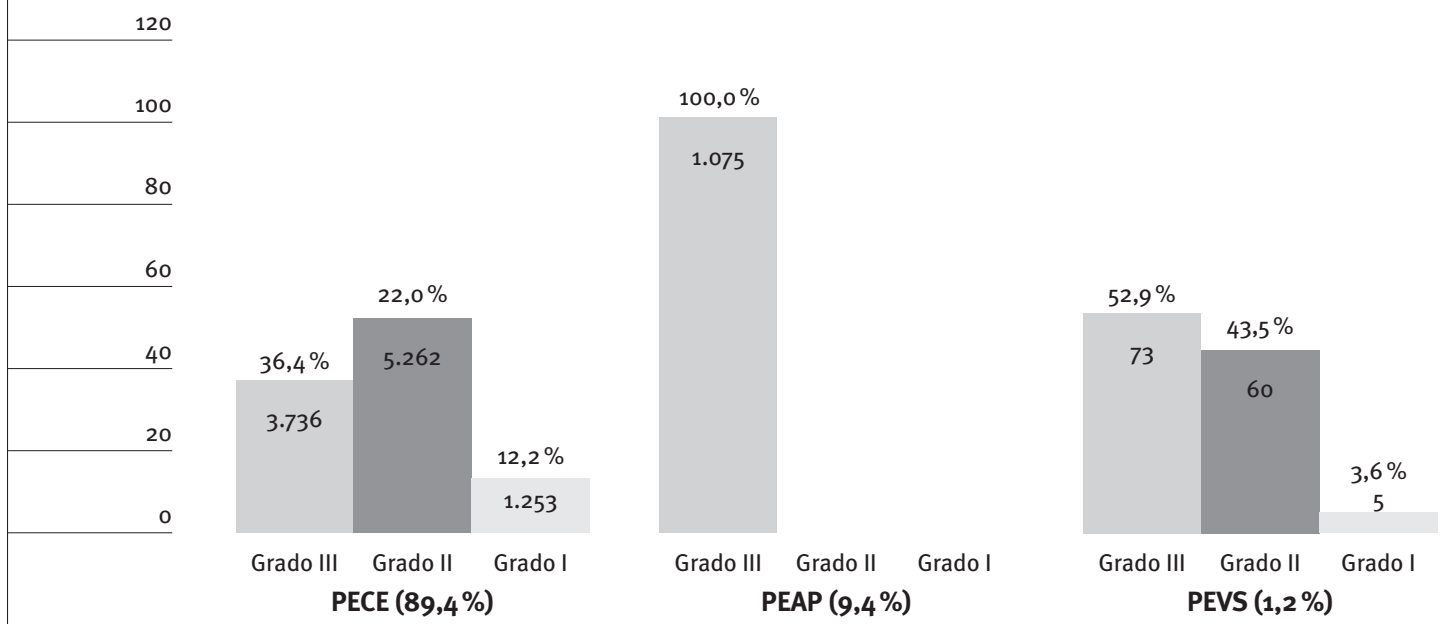

PECE: prestación para cuidados en el entorno familiar. PEAP: prestación de asistencia personal. PEVS: prestación vinculada a un servicio. Fuente: Elaboración propia a partir de datos del Departamento de Política Social de la Diputación Foral de Gipuzkoa.

El $51,5 \%$ de las personas perceptoras de la PECE eran dependientes de grado II; el 36,4\%, de grado III; y el $12,2 \%$, de grado I. La totalidad de las personas perceptoras de la PEAP eran dependientes de grado III (las únicas que en aquel momento tenían acceso a esta prestación). El 52,9\% de las personas perceptoras de la PEVS eran dependientes de grado III; el $43,5 \%$, de grado II; y el $3,5 \%$, de grado I.

La tasa de personas perceptoras de prestaciones económicas de dependencia con grado II era de 75,2 por cada 10.000 habitantes; la de las personas con grado III, de 69,1 por cada 10.000; y la de las personas con grado I, de 17,8 por cada 10.000. La tasa de personas percepto- ras de la PECE era de 144,9 por 10.000 habitantes; la de las perceptoras de la PEAP, de 15,2 por cada 10.000; y la de perceptoras de la PEVS, de 2,0 por cada 10.000 .

En función de la lógica intrínseca de la LAPAD, que planteaba las PECE con carácter de excepcionalidad 9 , lo normal habría sido que entre las personas dependientes de grado III fueran mayoritarias las PEAP ${ }^{10}$, y que las PECE tuvieran una presencia más residual. Sin embargo, en Gipuzkoa, como en el resto del Estado, no sucedió así. Esto se consideraba como una importante falta de previsión o adecuación de la Ley a la problemática real de la dependencia, sobre todo, en lo que respecta al colectivo de personas mayores.

Tabla 4. Distribución de los tipos de prestaciones económicas de dependencia, según el grado de dependencia de la persona beneficiaria. Gipuzkoa, septiembre de 2011

\begin{tabular}{|c|c|c|c|c|c|c|c|c|c|c|c|c|}
\hline \multirow[b]{3}{*}{ Prestaciones } & \multicolumn{9}{|c|}{ Grados de valoración de dependencia } & \multirow{2}{*}{\multicolumn{3}{|c|}{ Total }} \\
\hline & \multicolumn{3}{|c|}{ Grado III } & \multicolumn{3}{|c|}{ Grado II } & \multicolumn{3}{|c|}{ Grado I } & & & \\
\hline & Abs. & $\%$ & $\begin{array}{c}\text { Tasa por } \\
10.000 \\
\text { hab.* }\end{array}$ & Abs. & $\%$ & $\begin{array}{c}\text { Tasa por } \\
10.000 \\
\text { hab.* }\end{array}$ & Abs. & $\%$ & $\begin{array}{c}\text { Tasa por } \\
10.000 \\
\text { hab.* }\end{array}$ & Abs. & $\%$ & $\begin{array}{c}\text { Tasa por } \\
10.000 \\
\text { hab. }\end{array}$ \\
\hline PECE & 3.736 & 32,6 & 52,8 & 5.262 & 45,9 & 74,4 & 1.253 & 10,9 & 17,7 & 10.251 & 89,4 & 144,9 \\
\hline PEAP & 1.075 & 9,4 & 15,2 & - & - & - & - & - & - & 1.075 & 9,4 & 15,2 \\
\hline PEVS & 73 & 0,6 & 1,0 & 60 & 0,5 & 0,8 & 5 & - & 0,1 & 138 & 1,2 & 2,0 \\
\hline Total & 4.884 & 42,6 & 69,1 & $5 \cdot 322$ & 46,4 & 75,2 & 1.258 & 11,0 & 17,8 & 11.464 & 100,0 & 162,1 \\
\hline
\end{tabular}

PECE: prestación para cuidados en el entorno familiar. PEAP: prestación de asistencia personal. PEVS: prestación vinculada a un servicio. * La tasa se ha calculado sobre la población de Gipuzkoa registrada en el INE a 1 de enero del año correspondiente.

Fuente: Elaboración propia a partir de datos del Departamento de Política Social de la Diputación Foral de Gipuzkoa y el INE.

9 El artículo 14.4 de la LAPAD dice que: “El beneficiario podrá, excepcionalmente, recibir una prestación económica para ser atendido por cuidadores no profesionales, siempre que se den condiciones adecuadas de convivencia y de habitabilidad de la vivienda y así lo establezca su Programa Individual de Atención". Y el artículo 18.1 de la LAPAD apunta que: "Excepcionalmente, cuando el beneficiario esté siendo atendido por su entorno familiar, y se reúnan las condiciones establecidas en el artículo 14.4, se reconocerá una prestación económica para cuidados familiares".

${ }^{10}$ En 2011, la PEAP sólo era accesible a las personas con una valoración de dependencia de grado III. 
No obstante, en Gipuzkoa la PEAP tenía ya en 2011 más presencia que en ningún otro lugar del Estado, debido a una interpretación amplia del artículo 19 de la LAPAD, por la que se entendía que esta prestación económica, que tenía como objetivo contribuir a la contratación de una asistencia personal que facilitase al beneficiario el acceso a una vida más autónoma en el ejercicio de las actividades físicas de la vida diaria, también incluía, entre los potenciales beneficiarios, a las personas mayores de 65 años. En la actualidad, el mantenimiento de esta interpretación amplia del citado artículo, junto con la modificación legal previamente citada, está teniendo como consecuencia un incremento notable y progresivo en el número de dependientes que acceden a la PEAP; incremento que, en el resto del Estado, parece estar siendo mucho menor, con lo que la situación prácticamente no ha variado. Por otro lado, actualmente, la demanda de la PECE en Gipuzkoa muestra signos de haber 'tocado techo'.

\subsection{Recursos invertidos}

En el período comprendido entre 2008 y 2010 , la media anual de gasto en prestaciones económicas de dependencia fue de 30.666.953,33 euros, si bien anualmente este gasto varió de forma sensible. El primer año, ascendió a cerca de 19 millones de euros; en 2009 , se incrementó un 70,04\% y rebasó los 32 millones de euros; y en 2010, con un aumento del $26,57 \%$, alcanzó casi los 41 millones de euros. En tres años, la cantidad destinada a cubrir las prestaciones económicas de dependencia creció un 115,22\%. Este incremento medio fue aún mayor en lo relativo a las prestaciones para la asistencia personal (PEAP), pues alcanzó el 131,68\%; y fue muy inferior (36,63\%), en cambio, en las prestaciones vinculadas a la adquisición de un servicio (PEVS).

Del total de recursos utilizados anualmente, en torno al $70-80 \%$ ha correspondido a la prestación para cuidados en el entorno (PECE); alrededor de un $17-18 \%$, a la prestación de asistencia personal (PEAP); y entre el 2,3\% y el 4,6\%, a la prestación vinculada a la adquisición de un servicio (PEVS).

\subsection{Caracterización de las personas beneficiarias}

En septiembre de 2011 , el $\mathbf{2 1 , 1} \%$ de las personas perceptoras de prestaciones económicas de dependencia residían en la capital guipuzcoana; y un porcentaje algo inferior (el 18,9\%), en otros municipios de la comarca de Donostialdea. En Goierri, vivía el 13,1\% de las personas perceptoras; y en Urola-Kostaldea, el 12,8\%. En cada una de las restantes comarcas guipuzcoanas, residían entre el $7,4 \%$ y el $9,5 \%$ de las personas beneficiarias de estas prestaciones.

La distribución según comarcas de las personas perceptoras de la PECE se asemejaba mucho a la del conjunto; sin embargo, entre las personas receptoras de la PEAP y de la PEVS, se observaban diferencias. Así, entre las personas beneficiarias de la PEAP, la proporción de residentes en Donostia (30,0\%) y en Bidasoa Beherea (12,9\%) era mayor a la registrada en el conjunto del colectivo, e inferior, en cambio, el porcentaje de residentes en Donostialdea, Goierri, Tolosaldea y Urola-Kostaldea. Entre las personas beneficiarias de la PEVS, la proporción de residentes en Donostia, en Donostialdea y en Bidasoa Beherea era mayor que la observada en el conjunto, y en cambio, inferior la de los residentes en el resto de las comarcas.

Gráfico 4. Evolución del gasto en prestaciones de la Ley de Dependencia. Gipuzkoa, 2008-2010 (euros)

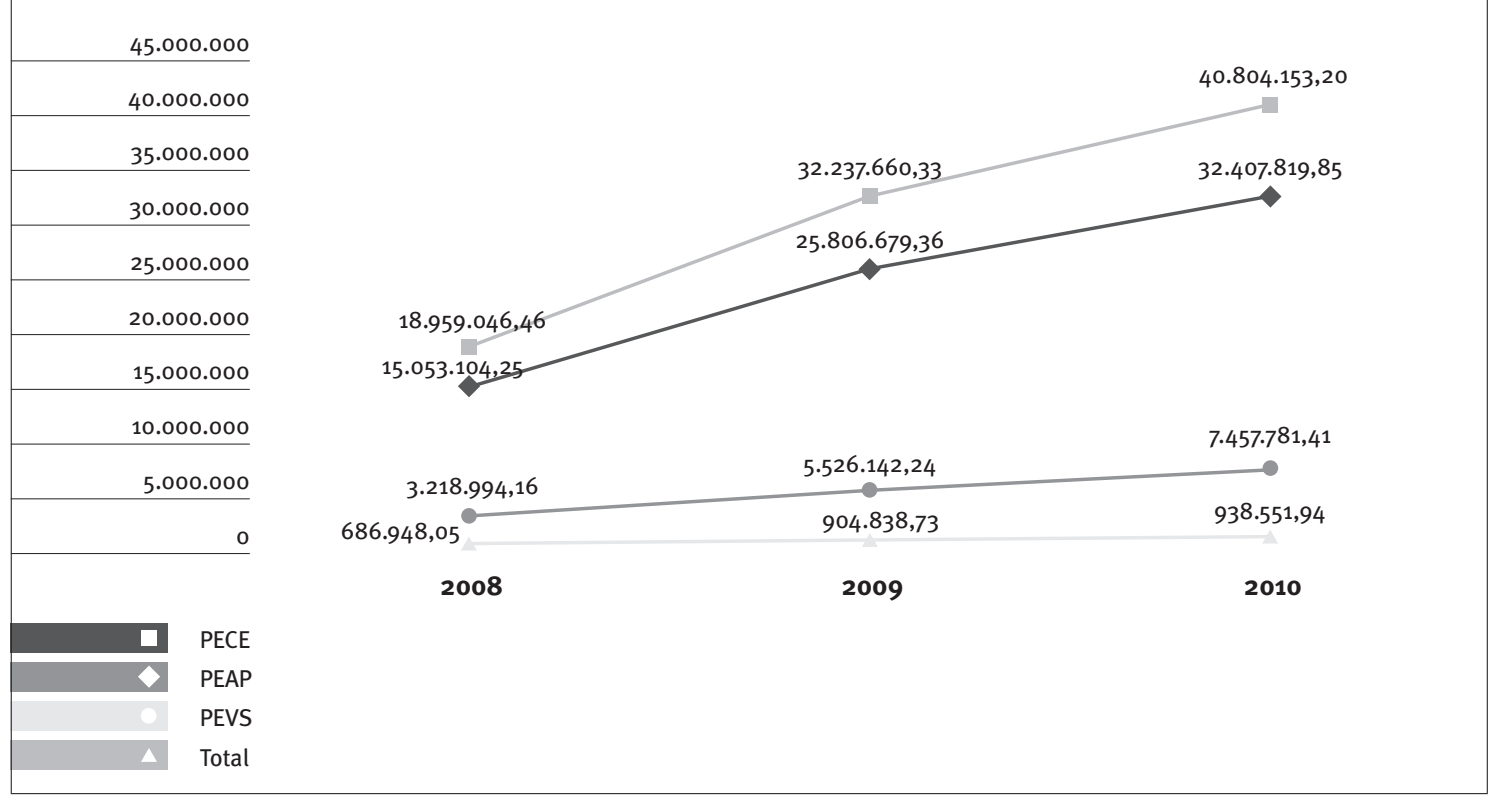

PECE: prestación para cuidados en el entorno familiar. PEAP: prestación de asistencia personal. PEVS: prestación vinculada a un servicio. Fuente: Elaboración propia a partir de datos del Departamento de Política Social de la Diputación Foral de Gipuzkoa. 
Cruzando ambas variables, observamos que el $18 \%$ del conjunto de las personas perceptoras de prestaciones de dependencia correspondían a perceptoras de la PECE residentes en Donostia; el 17,4\%, a perceptoras de la PECE residentes en Donostialdea; en torno al $12 \%$, a perceptoras de la PECE residentes en Goierri; y otro $11,8 \%$, a perceptoras de la PECE residentes en Urola-Kostaldea.

La incidencia o tasa de personas perceptoras por cada 10.000 habitantes variaba sensiblemente según la comarca: alcanzaba sus mayores valores en Goierri, Urola-Kostaldea y Deba Beherea (221,1, 202,7 y 197,1, respectivamente) e iba descendiendo hasta 130,6 en el caso de Donostia y 119,4 en Bidasoa Beherea.

En relación a la edad de las personas perceptoras de prestaciones económicas de dependencia en Gipuzkoa, se observa que más de la mitad (el $55,8 \%$, es decir, 6.392 personas) contaba con más de 80 años; algo menos de la cuarta parte (el 23,6\%, o sea, 2.707 personas), entre 60 y 79 años; el $15 \%$, entre 18 y 59 años (1.715 personas); y casi el $6 \%$, eran niños/as y jóvenes menores de 18 años (650 personas).
La distribución por edad de las personas perceptoras de la PECE se asemejaba bastante a la tendencia general previamente señalada, si bien el porcentaje de personas mayores de 80 años era ligeramente inferior (52,8\%), y ligeramente superior, en cambio, el peso de los otros tres grupos de edad. Entre las personas perceptoras de la PEAP y de la PEVS, el peso relativo de las mayores de 80 años era muy superior, y alcanzaba el $80,6 \%$ en el caso de la PEAP y el $85,5 \%$ en el de la PEVS.

Casi la mitad del colectivo de personas perceptoras de estas prestaciones económicas estaba integrado por mayores de 80 años que percibían la PECE (47,2\%); y otro $22,1 \%$ lo constituían las personas perceptoras de la PECE de entre 60 y 79 años. Por cada 10.000 habitantes de Gipuzkoa, 162,1 percibían alguna prestación económica de dependencia en 2011, una tasa que variaba sensiblemente según el estrato de edad analizado. Así, entre la población mayor de 80 años, 1.621,3 de cada 10.000 recibían alguna prestación (la gran mayoría de ellas, la PECE); entre la población de 60 a 79 años, la tasa era de 193,7; entre la población menor de 18 años, de 55,8; y entre la población de 18 a 59 años, de 41,7.

Tabla 5. Comarca de residencia de las personas perceptoras de prestaciones económicas de dependencia. Gipuzkoa, 2011

\begin{tabular}{|c|c|c|c|c|c|c|c|c|c|c|c|c|c|c|c|}
\hline \multirow{3}{*}{$\begin{array}{l}\text { Comarca de } \\
\text { residencia }\end{array}$} & \multicolumn{12}{|c|}{ Prestaciones de dependencia } & \multirow{2}{*}{\multicolumn{3}{|c|}{ Total }} \\
\hline & \multicolumn{4}{|c|}{$\begin{array}{c}\text { Para cuidados en el entorno } \\
\text { familiar (PECE) }\end{array}$} & \multicolumn{4}{|c|}{ De asistencia personal (PEAP) } & \multicolumn{4}{|c|}{$\begin{array}{c}\text { Vinculadas a la adquisición } \\
\text { de un servicio (PEVS) }\end{array}$} & & & \\
\hline & Abs. & $\%$ & $\begin{array}{c}\% \\
\text { (total de } \\
\text { respuestas) }\end{array}$ & \begin{tabular}{|c|} 
Tasa \\
por \\
10.000 \\
hab.* \\
\end{tabular} & Abs. & $\%$ & $\begin{array}{c}\% \\
\text { (total de } \\
\text { respuestas) }\end{array}$ & \begin{tabular}{|c|} 
Tasa \\
por \\
10.000 \\
hab.* \\
\end{tabular} & Abs. & $\%$ & $\%$ resp. & $\begin{array}{l}\text { Tasa } \\
\text { por } \\
1.000 \\
\text { hab.* }\end{array}$ & Abs. & $\%$ & \begin{tabular}{|c|} 
Tasa \\
por \\
10.000 \\
hab.* \\
\end{tabular} \\
\hline $\begin{array}{l}\text { Bidasoa } \\
\text { Beherea }\end{array}$ & 753 & 7,3 & 6,6 & 97,3 & 139 & 12,9 & 1,2 & 18,0 & 32 & 23,2 & 0,3 & 4,1 & 924 & 8,1 & 119,4 \\
\hline Deba Beherea & 969 & 9,5 & 8,5 & 176,2 & 108 & 10,0 & 0,9 & 19,6 & 7 & 5,1 & 0,1 & 1,3 & 1.084 & 9,5 & 197,1 \\
\hline Deba Garaia & 943 & 9,2 & 8,2 & 152,4 & 99 & 9,2 & 0,9 & 16,0 & 7 & 5,1 & 0,1 & 1,1 & 1.049 & 9,2 & 169,6 \\
\hline Donostialdea & 1.995 & 19,5 & 17,4 & 142,9 & 144 & 13,4 & 1,3 & 10,3 & 29 & 21,0 & 0,3 & 2,1 & 2.168 & 18,9 & 155,3 \\
\hline Donostia & 2.065 & 20,1 & 18,0 & 111,3 & 322 & 30,0 & 2,8 & 17,4 & 36 & 26,1 & 0,3 & 1,9 & 2.423 & 21,1 & 130,6 \\
\hline Goierri & 1.385 & 13,5 & 12,1 & 203,8 & 105 & 9,8 & 0,9 & 15,5 & 12 & 8,7 & 0,1 & 1,8 & 1.502 & 13,1 & 221,1 \\
\hline Tolosaldea & 783 & 7,6 & 6,8 & 165,2 & 56 & 5,2 & 0,5 & 11,8 & 5 & 3,6 & 0,0 & 1,1 & 844 & 7,4 & 178,0 \\
\hline $\begin{array}{l}\text { Urola- } \\
\text { Kostaldea }\end{array}$ & 1.358 & 13,2 & 11,8 & 187,2 & 102 & 9,5 & 0,9 & 14,1 & 10 & 7,2 & 0,1 & 1,4 & 1.470 & 12,8 & 202,7 \\
\hline Total & 10.251 & 100,0 & 89,4 & 144,9 & 1.075 & 100,0 & 9,4 & 15,2 & 138 & 100,0 & 1,2 & 2,0 & 11.464 & 100,0 & 162,1 \\
\hline
\end{tabular}

* La tasa por cada 10.000 habitantes está calculada sobre la población de cada una de las comarcas.

Fuente: Elaboración propia a partir de datos del Departamento de Política Social de la Diputación Foral de Gipuzkoa.

Tabla 6. Edad de las personas perceptoras de prestaciones económicas de dependencia. Gipuzkoa, 2011

\begin{tabular}{|c|c|c|c|c|c|c|c|c|c|c|c|c|c|c|c|}
\hline \multirow[b]{3}{*}{ Universo } & \multicolumn{12}{|c|}{ Prestaciones de dependencia } & \multirow{2}{*}{\multicolumn{3}{|c|}{ Total }} \\
\hline & \multicolumn{4}{|c|}{$\begin{array}{l}\text { Para cuidados en el entorno } \\
\text { familiar (PECE) }\end{array}$} & \multicolumn{4}{|c|}{ De asistencia personal (PEAP) } & \multicolumn{4}{|c|}{\begin{tabular}{|c|}
$\begin{array}{c}\text { Vinculadas a la adquisición de un } \\
\text { servicio (PEVS) }\end{array}$ \\
\end{tabular}} & & & \\
\hline & Abs. & $\%$ & $\begin{array}{c}\% \text { (total de } \\
\text { respuestas) }\end{array}$ & $\begin{array}{c}\text { Tasa } \\
\text { por } \\
10.000 \\
\text { hab.* }\end{array}$ & Abs. & $\%$ & $\begin{array}{c}\% \text { (total de } \\
\text { respuestas) }\end{array}$ & $\begin{array}{c}\text { Tasa } \\
\text { por } \\
10.000 \\
\text { hab.* }\end{array}$ & Abs. & $\%$ & $\begin{array}{c}\% \text { (total de } \\
\text { respuestas) }\end{array}$ & $\begin{array}{c}\text { Tasa } \\
\text { por } \\
1.000 \\
\text { hab.* }\end{array}$ & Abs. & $\%$ & $\begin{array}{c}\text { Tasa } \\
\text { por } \\
10.000 \\
\text { hab.* }\end{array}$ \\
\hline$>80$ años & 5.408 & 52,8 & 47,2 & $1.371,1$ & 866 & 80,6 & 7,6 & 219,7 & 118 & 85,5 & 1,0 & 29,9 & 6.392 & 55,8 & $1.621,3$ \\
\hline 60-79 años & 2.534 & 24,7 & 22,1 & 181,3 & 153 & 14,2 & 1,3 & 10,9 & 20 & 14,5 & 0,2 & 1,4 & 2.707 & 23,6 & 193,7 \\
\hline 18-59 años & 1.673 & 16,3 & 14,6 & 40,7 & 42 & 3,9 & 0,4 & 1,0 & - & - & - & - & 1.715 & 15,0 & 41,7 \\
\hline 0-17 años & 636 & 6,2 & 5,5 & 54,6 & 14 & 1,3 & 0,1 & 1,2 & - & - & - & - & 650 & 5,7 & 55,8 \\
\hline Total & 10.251 & 100,0 & 89,4 & 144,9 & 1.075 & 100,0 & 9,4 & 15,2 & 138 & 100,0 & 1,2 & 2,0 & 11.464 & 100,0 & 162,1 \\
\hline
\end{tabular}

* La tasa por cada 10.000 habitantes está calculada sobre la población de cada uno de los grupos de edad.

Fuente: Elaboración propia a partir de datos del Departamento de Política Social de la Diputación Foral de Gipuzkoa. 


\section{La concurrencia entre prestaciones económicas y servicios sociales}

\subsection{Modelo de atención a la dependencia}

El modelo de atención a la dependencia de Gipuzkoa se ha basado en la posibilidad de combinar servicios sociales y prestaciones económicas como forma de lograr una respuesta óptima a las necesidades de la persona dependiente. Con la puesta en marcha de la LAPAD, se ha constatado un desarrollo descompensado de las prestaciones económicas y de los servicios sociales: se han desarrollado mucho las primeras y no así los servicios previamente existentes (servicio de ayuda a domicilio, centros de día, residencias), ni otros nuevos susceptibles de creación (centros de noche, servicio de lavandería). Esto no parece acorde con el espíritu de la propia Ley, que considera que los servicios de prevención de las situaciones de dependencia y los de promoción de la autonomía personal tendrán un carácter prioritario frente a las prestaciones económicas. En este sentido, puede decirse que los servicios sociales precisan de un nuevo impulso o desarrollo, que permita ajustarlos a las nuevas problemáticas emergentes en relación con la dependencia. Ello posibilitaría que ambas líneas de trabajo social (prestaciones y servicios) pudieran tener un mayor grado de complementariedad y acompasamiento.

Según datos de junio de 2010, el conjunto de personas beneficiarias de prestaciones económicas de dependencia (9.147 personas) disfrutaban de 5.147 servicios, es decir, que había un ratio de 0,56 servicios por persona. El servicio más extendido era el relacionado con las ayudas técnicas, del que disfrutaba el $28,6 \%$ de las personas beneficiarias; a continuación se situaba el servicio de asistencia domiciliaria, utilizado por el 13,9\%; y en tercer lugar, el centro de día para personas mayores (9,1\%). Los centros de día para personas con discapacidad eran utilizados por el 2,1\%; los talleres protegidos para personas con discapacidad, por el 1,8\%; y los restantes servicios (residencias para personas mayores, residencias para personas con discapacidad, centros de día de atención a la salud mental y centros sociosanitarios), por menos del $1 \%$.

Las personas perceptoras de la PECE constituían la gran mayoría del colectivo de personas beneficiarias de algún tipo de prestación económica, concretamente el $88 \%$. Ello hacía que los datos relativos a los servicios que utilizaban fuesen muy similares a los que acabamos de comentar: así, contaban con un ratio de 0,56 servicios por persona beneficiaria, siendo los servicios más extendidos los relativos a las ayudas técnicas (27,2\%), seguidas del servicio de asistencia domiciliaria (que utilizaba el 14,0\%) y del centro de día para personas mayores (9,6\%). Los centros de día para personas con discapacidad eran utilizados por el 2,3\% de personas beneficiarias y el $2,1 \%$ de ellas acudían a los talleres protegidos para personas con discapacidad.

Las personas perceptoras de la PEAP representaban el $10,5 \%$ del conjunto de personas beneficiarias, y su ratio de servicios por persona era ligeramente superior al del conjunto $(0,65)$. En este grupo, era

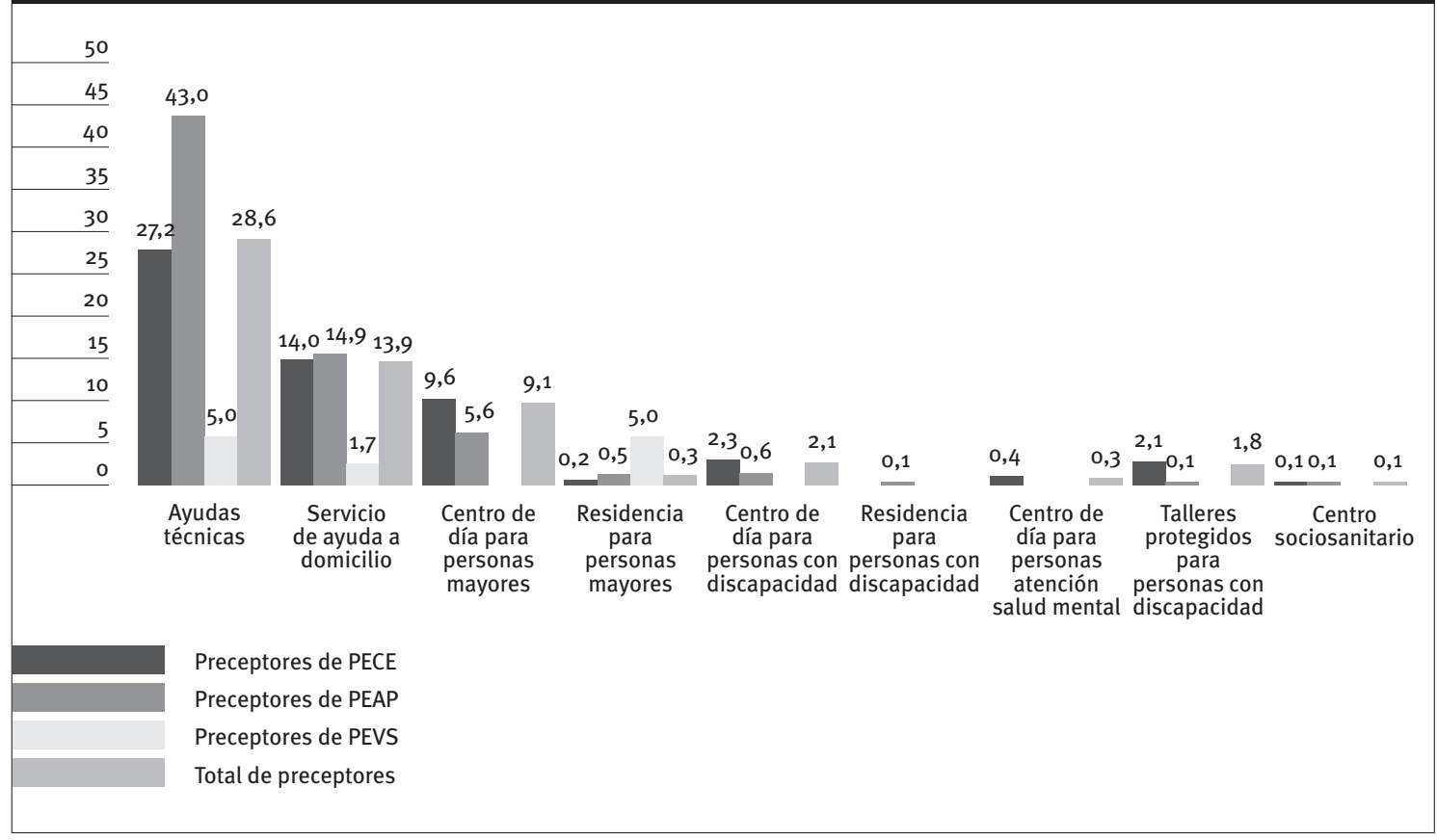

PECE: prestación para cuidados en el entorno familiar. PEAP: prestación de asistencia personal. PEVS: prestación vinculada a un servicio. Fuente: Elaboración propia a partir de datos del Departamento de Política Social de la Diputación Foral de Gipuzkoa. 
sensiblemente mayor el porcentaje de personas beneficiarias que disfrutaban de ayudas técnicas ( $43 \%)$, y menor, en cambio, el porcentaje de personas que recurrían a centros de día para personas mayores $(5,6 \%)$, centros de día para personas con discapacidad (o,6\%) y a los talleres protegidos para personas con discapacidad $(0,1 \%)$.

Las personas beneficiarias de la PEVS ${ }^{11}$ representaban tan sólo el 1,3\% del conjunto, y apenas recurrían a servicios (el ratio de servicios/persona era de 0,12$)$. En este caso, los más habituales eran las ayudas técnicas, utilizadas por el $5 \%$ de las personas beneficiarias.

\subsection{Visión de la concurrencia entre prestaciones y servicios desde la perspectiva de los agentes de intervención}

La vinculación directa del derecho a la prestación económica con el reconocimiento de la existencia de dependencia (como derecho subjetivo) ha permitido que pueda solicitarse independientemente del tipo de prestación (social o económica) que, desde un punto de vista técnico-profesional, se considere más adecuada a las necesidades de la persona dependiente.

En algunos casos, en la práctica, las prestaciones económicas de dependencia han estado sirviendo como fuente de ingresos complementarios para hacer frente a los gastos del hogar (más aún en el contexto de crisis económica). Y en la medida en que la utilización de los servicios sociales a la dependencia conlleva una disminución en la cuantía de las prestaciones económicas, se ha observado que ello supone un freno a la demanda de tales servicios.

Las condiciones económicas de acceso a las distintas prestaciones del sistema de dependencia poseen un tratamiento diferenciado: mientras que las prestaciones económicas son accesibles a la práctica totalidad del colectivo de personas dependientes (por la escasa incidencia de la situación económicopatrimonial de las personas beneficiarias a la hora de condicionar el acceso a estas ayudas), el recurso a los servicios sociales está condicionado por el coste económico (copago) que suponen, dependiendo de la situación económica de la persona dependiente y su entorno familiar. Así, a menor coste de los servicios sociales, se observa una mayor tendencia a su mantenimiento. Esto explica la menor incidencia de las prestaciones económicas en el colectivo de personas que ya eran usuarias del servicio de asistencia domiciliaria: los colectivos que lo utilizan, en general, cuentan con un nivel socioeconómico bajo, muy reducido para ellos.

${ }^{11}$ Las personas dependientes que perciben una prestación vinculada al servicio apenas acceden a otros servicios, debido a que es el centro residencial en el que ingresan el que cubre sus necesidades. En algunos casos, siguen de alta en ayudas técnicas, porque mantienen, por ejemplo, las sillas de ruedas eléctricas.
Entre los agentes de intervención, se ha identificado un discurso que señala que el freno en la demanda de servicios sociales detectado a partir de la puesta en marcha de las prestaciones económicas puede estar denotando una disfunción importante en el funcionamiento del sistema para la autonomía y atención a la dependencia, por cuanto se está produciendo una sustitución de servicios profesionalizados, como el servicio de asistencia domiciliaria, por cuidados no profesionalizados que no garantizan los mismos niveles de calidad para la atención y autonomía de la persona dependiente.

Con la puesta en marcha de las prestaciones económicas, se ha generado un desequilibrio entre prestaciones y servicios sociales: se han destinado muchos recursos a las prestaciones económicas, mientras que los destinados a los servicios sociales se han mantenido estancados. Esto ha dificultado el desarrollo de una correcta labor de orientación de los servicios sociales de base, debido a la carencia de una carta de servicios ajustada a las diversas situaciones sociales y económicas que presenta un colectivo tan heterogéneo y amplio como el de las personas dependientes. Teniendo en cuenta que la propia Ley prioriza los servicios sociales frente a las prestaciones, se considera fundamental el desarrollo de los primeros. Ello, por un lado, va a permitir mejorar la calidad de atención a las personas dependientes y, por otro, va a ser una inversión a medio plazo, en la medida en que posibilitaría ralentizar procesos de deterioro de personas dependientes, evitando o retrasando la demanda de recursos residenciales más costosos.

Por otra parte, también se ha detectado la ausencia de una coordinación sistemática y estable entre los recursos sociales y sanitarios a escala local, lo que impide la conformación y articulación de un espacio sociosanitario de base a partir del que identificar potenciales dependientes y desarrollar servicios de atención sociosanitaria domiciliaria de carácter preventivo, orientados no tanto a la atención de la persona dependiente, sino a la promoción de su autonomía en fases iniciales. Ello permitiría prolongar la permanencia de las personas dependientes en su entorno familiar y ralentizar o disminuir los ingresos en recursos residenciales, con el consiguiente ahorro de costes.

Además de esto, también se ha señalado la existencia de un conocimiento insuficiente o difuso del catálogo de servicios sociales por parte de diversos colectivos de usuarios/as potenciales, que condiciona su interés y predisposición al uso de tales servicios.

\subsection{La concurrencia entre prestaciones y servicios desde la perspectiva de las familias}

Entre las familias perceptoras de prestaciones económicas de dependencia, la necesidad de ambos tipos de prestación (económica y de servicios sociales) aparece sobre todo cuando existen grandes dependientes supervisados (no necesariamente atendidos) 
por familiares de otra generación (hijos/as, nueras, sobrinos/as) que priorizan el mantenimiento en el entorno familiar, para lo que requieren asistencia personal continuada (PEAP), combinada con otros servicios sociales (asistencia domiciliaria o centro de día), que den apoyo bien a personas cuidadoras de edad muy avanzada con limitada capacidad de atención, bien a cuidadores más jóvenes para que puedan desarrollar una vida más normalizada (actividad laboral, relación social, salidas y encargos). Estas familias han recalcado la importancia de la prestación integral y combinada de servicios sociales y apoyos económicos, considerando que ambos elementos son compatibles y necesarios para atender las necesidades de la persona dependiente, así como para permitir el desarrollo de una vida normalizada por parte de los familiares a su cargo.

La priorización de los servicios sociales frente a la prestación económica, en gran medida, parece depender de la situación económica de la familia. Así, a peor situación económica, la familia otorga mayor prioridad a la percepción de una prestación económica; $y$, al revés, a mejor situación económica de la familia, más valora la posibilidad de acceder a unos servicios sociales adecuados.

En general, las familias han priorizado los servicios frente a las prestaciones debido a las siguientes razones:

- Los servicios sociales (sobre todo, el centro de día) son un recurso básico que permite el respiro y el desarrollo de una vida más normalizada por parte de las familias y de las personas cuidadoras en particular, más aún en el caso de personas con alto grado de dependencia que requieren de un apoyo intenso y continuado. En este sentido, es un recurso muy valorado por personas cuidadoras no mayores que, como ya se ha señalado, necesitan apoyos para poder desarrollar una vida normalizada.

- Los servicios sociales tienen una incidencia directa en la mejora de la atención y cuidados que recibe la persona dependiente, mientras que el destino de las prestaciones económicas (PECE) queda en manos de los/as perceptores/as o sus familiares.

- Los servicios sociales tienen un componente de dinamización de la persona dependiente y de relación social que promueve su autonomía personal.

- En el caso de municipios pequeños o relativamente aislados, se precisa garantizar la accesibilidad a recursos sociales (centro de día, talleres ocupacionales, asociaciones) que permitan a la familia desarrollar una vida relativamente normalizada, sin que sea precisa la atención permanente por parte algún miembro del hogar.

La PECE se ha mostrado como el recurso más adecuado y valorado entre quienes consideran que la mejor atención se proporciona en el entorno familiar, y también cuando la persona cuidadora está dispuesta y se siente capacitada para responder a las necesidades de la persona dependiente. Desde este planteamiento, los familiares consideran que, en el proceso evolutivo de la atención, el acceso a los servicios sociales se debe producir en el momento en que no es posible atender adecuadamente a la persona en el hogar. Hasta ese momento, priorizan la prestación económica.

Se ha observado que la opción por la prestación económica se fundamenta, más que en su funcionalidad directa, en la escasa valoración o el 'rechazo' de los servicios sociales existentes. Así, entre las familias analizadas, se han detectado situaciones como:

- Un fuerte recelo a delegar el cuidado de la persona dependiente en terceras personas 0 instituciones (por ruptura de vínculo afectivo familiar) y un rechazo a permitir la entrada en el hogar a terceras personas (sentimiento de injerencia). Este rechazo parece más habitual entre las personas cuidadoras de más edad que entre las más jóvenes, así como en familiares con un componente cultural muy arraigado de asunción natural de la responsabilidad de cuidar a los/as familiares, incluso cuando se trata de cuidados intergeneracionales.

- Pese a la prioridad que algunos miembros de la familia otorgan a los servicios sociales, se opta por las prestaciones económicas como resultado del rechazo a recurrir a los servicios sociales por parte de la persona cuidadora o de la propia persona dependiente. Cuando este rechazo proviene sólo de la persona dependiente y es aceptado por la cuidadora, de alguna forma, ésta puede tener una vivencia de 'cautividad' o 'aislamiento' que tampoco parece beneficiar a la persona dependiente desde el punto de vista de la promoción de la actividad y las relaciones sociales.

- Una imagen negativa de los servicios sociales (sobre todo, los que tienen un componente de residencialización), debido a una percepción de carencia de un adecuado trato afectivo y personalizado hacia las personas usuarias. Esta imagen básicamente se asocia a aquellos recursos sociales dirigidos a las personas mayores.

Por otro lado, se ha constatado que, entre las familias perceptoras de la PEAP, existe una tendencia general a priorizar la prestación frente a los servicios, debido a:

- La consideración de que la PEAP garantiza la posibilidad de contar con una asistencia personal continuada en el propio entorno familiar, siendo éste el recurso que se considera prioritario tanto para la persona dependiente como para sus familiares cuidadores.

- El rechazo de la familia a la salida de la persona dependiente del domicilio, por considerar que tan importante como la atención y cuidados es el trato afectivo y el mantenimiento del vínculo físico familiar. 
- La ausencia de recursos sociales (sobre todo, el centro de día) que respondan adecuadamente a las necesidades de grandes dependientes o de dependientes con problemas específicos (por ejemplo, demencias), lo que hace que la PEAP sea considerada como la prestación que en mayor medida responde a las necesidades tanto de las personas dependientes como de sus cuidadoras familiares para que puedan desarrollar una vida lo más normalizada posible.

\subsection{Primacía de las prestaciones económicas frente a los servicios}

Cualitativamente, se ha detectado que las principales motivaciones que contribuyen a explicar el gran desarrollo y extensión de las prestaciones económicas frente al estancamiento en el desarrollo de los servicios sociales son las siguientes:

1. La concepción de derecho subjetivo de las prestaciones económicas: el reconocimiento de un determinado grado y nivel de dependencia lleva implícito el derecho a percibir una prestación económica (no se exige ninguna condición específica). Por tanto, un segmento significativo del colectivo de potenciales dependientes sólo acude a los servicios sociales de base con el objeto expreso de acceder a las prestaciones económicas (se puede decir que, en gran medida, es un contacto de carácter básicamente administrativo).

2. La carestía de los servicios sociales (competencia en el mercado) y las fórmulas de copago establecidas para el acceso a ellos. De hecho, se considera que el sistema de copago que pueda plantearse en el futuro puede ser uno de los factores determinantes del éxito o fracaso de posibles nuevos servicios o recursos.

3. La falta de capacidad prescriptora de los servicios sociales de base en torno al diagnóstico de la situación de dependencia (por ausencia de apoyo normativo; por creencia social de las familias, que frecuentemente no les reconocen esta función).

4. El carácter asistencialista de los servicios, centrado en la atención y el cuidado, y alejado del derecho subjetivo que promueve la autonomía personal.

5. La escasez e inadecuación de los servicios frente a las problemáticas específicas de dependencia: estancamiento, falta de desarrollo (por ejemplo, plazas en centros de día o residencias, recursos para dependientes con discapacidad intelectual, servicios formativos).

6. La flexibilidad en el uso de las prestaciones económicas por parte de las familias (se solicitan escasas contraprestaciones y no hay un excesivo control) frente a la regulación y rigidez de la oferta de los servicios (como la limitación del servicio de asistencia domiciliaria a un máximo tres horas).
7. El arraigo cultural del cuidado y atención a los familiares dependientes en el entorno familiar, con 'rechazo' de la presencia e intervención de terceras personas en el hogar (cuidadores externos a través de la PEAP, asistentes sociales a través del servicio de asistencia domiciliaria). Esta cultura presenta una mayor incidencia en entornos rurales en los que, tradicionalmente, la responsabilidad del cuidado ha recaído en la figura de la mujer (hija, madre o esposa).

\section{Impacto de las prestaciones económicas de dependencia}

\subsection{Consideraciones generales sobre el funcionamiento de las prestaciones}

La carencia de mecanismos de seguimiento sistemático ha impedido conocer objetivamente el impacto real de las prestaciones en la mejora de la calidad de atención y cuidados a las personas dependientes. Existe un discurso que señala la necesidad de evaluar el uso y el impacto de estas prestaciones. En todo caso, nos parece importante apuntar las siguientes consideraciones que, en relación al funcionamiento de estas prestaciones, hemos comenzado a detectar:

- Las prestaciones económicas posibilitan el mantenimiento de las personas dependientes en el entorno familiar, respondiendo así al deseo mayoritario de las personas dependientes, que desean continuar en su hogar. Desde la perspectiva de los servicios sociales, la prestación económica (y el consiguiente mantenimiento en el hogar) tiene como consecuencia 'apaciguar' las demandas y solicitudes de la ciudadanía ante la escasez de oferta o la carestía de distintos servicios sociales, y retrasar la solicitud de ingreso en recursos asistenciales, económicamente más costosos y a los que mayormente se acude cuando el grado de deterioro es ya muy elevado.

- Las prestaciones económicas han contribuido a hacer socialmente visible la situación de dependencia, lo cual ha facilitado a los servicios sociales realizar el seguimiento e intervenir en situaciones que antes estaban 'fuera del sistema'.

- Las prestaciones económicas de dependencia se ven reducidas considerablemente, en ocasiones hasta tener un carácter casi residual o simbólico, en el caso de colectivos que, por la especificidad de su enfermedad o discapacidad, ya están accediendo a otro tipo de recursos (centro de día, taller ocupacional), o están recibiendo otras ayudas o prestaciones económicas.

- Las prestaciones económicas actuales están dejando al margen a un colectivo de personas especialmente vulnerable, como el de las personas mayores que viven solas, bien por su desconocimiento sobre el sistema de prestaciones, bien por no cumplir las condiciones exigidas: residen solas (por tanto, no pueden acceder a la PECE), no llegan 
al grado III (y no pueden acceder a la PEAP), o carecen de recursos para acceder a una residencia privada (y, por tanto, no pueden acceder a la PEVS).

Por otra parte, se señala la importancia de introducir mecanismos que permitan mejorar la calidad de la atención que reciben las personas dependientes, tales como: una mayor formación de las personas cuidadoras, un mayor acceso a servicios sociales profesionalizados (a los que, frecuentemente, la familia o la propia persona dependiente no desean recurrir). Para ello, se apunta la conveniencia de incluir o acordar contraprestaciones/compromisos como condición para acceder a prestaciones económicas (en materia de formación de la persona cuidadora, de utilización de determinados recursos sociales).

\subsection{El impacto de la prestación económica vinculada a la adquisición de un servicio (PEVS)}

La PEVS se ha concebido como una solución de tránsito hasta la consecución de la plaza en un centro residencial público o concertado, si bien restringida a una demanda con cierto poder adquisitivo, que cuente con recursos económicos para poder hacer frente al coste de una plaza residencial privada, ya que la PEVS suele cubrir una escasa parte de dicho coste. Esto, unido al interés de muchas familias por retrasar al máximo el ingreso del familiar dependiente en un recurso residencial, ha hecho que el número de prestaciones de este tipo en Gipuzkoa sea realmente reducido.

El planteamiento de una hipotética reorientación de la PEVS como recurso económico para poder acceder a asistencia de carácter domiciliario no se considera adecuado, en la medida en que esta asistencia domiciliaria se corresponde a grados de dependencia y de necesidad inferiores a la de quien precisa un recurso residencial: cuando se opta por la PEVS, normalmente se debe a que la familia de la persona dependiente considera que ningún otro servicio puede responder o reemplazar la necesidad de residencialización; de alguna forma, es el último recurso, al que tan sólo se accede tras haber agotado el resto de alternativas posibles.

\subsection{El impacto de la prestación económica de asistencia personal (PEAP)}

Una de las funcionalidades básicas de esta prestación ha sido la regularización o aumento en la dedicación de una tercera persona que, previamente a la concesión de la prestación, ya se ocupaba de los cuidados y atención de la persona dependiente. La condición de regularizar laboralmente a las personas que prestan la asistencia personal ha podido retraer el acceso a esta prestación en casos en los que, previamente, se contaba con los servicios de una tercera persona de confianza a la que se desea dar continuidad, pero manteniendo su situación irregular (por ejemplo, en el caso de inmigrantes).
Ha resultado bastante evidente que la realidad no ha respondido al espíritu de la Ley en cuanto a la prioridad que se trataba de otorgar a las PEAP respecto a las $\mathrm{PECE}$, con lo que parece que no se ha contribuido demasiado a ofertar servicios profesionales y especializados en los hogares de las personas dependientes y, por tanto, la generación de empleo regularizado en torno a la actividad de atención y cuidados profesionales a las personas dependientes ha resultado muy escasa respecto a las previsiones iniciales.

En general, a la hora de valorar esta prestación, las familias beneficiarias han destacado los siguientes aspectos:

- Se considera como un apoyo económico para la contratación, regularización o prolongación de un servicio que se considera básico, más aún en el caso de familias con situación económica precaria.

- Supone un respiro y un apoyo para que los familiares puedan desarrollar una vida más normalizada (actividad laboral, relaciones sociales), así como para descargar la responsabilidad de la atención, sobre todo, en el caso de personas cuidadoras mayores con limitaciones para desarrollar esta función.

Son también las familias las que han planteado la insuficiencia de la cuantía de la prestación en contextos familiares de gran dependencia (más de un miembro dependiente), con necesidad intensa y prolongada de atención (que a veces requiere la contratación de más de un asistente personal).

Algunos agentes de intervención o profesionales de los servicios sociales han señalado la conveniencia de impulsar en mayor medida la PEAP y en menor medida la PECE, por cuanto esta última es un tipo de prestación que, en principio, tiende a favorecer el cuidado familiar no profesionalizado, menos controlable y de carácter excepcional (según señala la Ley). Sin embargo, en la práctica, la PEAP no ha garantizado la prestación de un nivel de atención y cuidados superior a la PECE, debido a que no ha habido una exigencia de formación o competencias especiales para las y los cuidadores contratados a través de la PEAP.

No obstante, también se ha constatado que la asistencia personal tiene un componente más profesionalizado en los casos en los que las personas cuidadoras proceden de estructuras organizativas o empresas de servicios que les dotan de cierta formación. Ello implica una mejora en la calidad de la atención y cuidados prestados. En cualquier caso, cabe destacar el escaso grado de concreción formal de la cualificación precisa para garantizar un servicio de asistencia personal profesionalizado. 


\subsection{El impacto de la prestación económica para cuidados en el entorno familiar y apoyo a cuidadores no profesionales (PECE)}

La PECE se considera un apoyo más o menos importante para la familia en función de su propia situación económica. Así se puede vivenciar como:

- un ingreso complementario, en familias con una situación económica normalizada, sin que suponga un impacto significativo en la situación previa a su recepción; 0

- un ingreso importante para hacer frente a los gastos familiares en general, especialmente en el caso de familias con una economía precaria (personas jubiladas, pensionistas, personas desempleadas).

Las familias han señalado que los principales destinos hacia los que se dirige la PECE son:

- El pago de profesionales y especialistas para una mejor atención y promoción de la autonomía de la persona dependiente: homeópatas, logopedas, podólogos, osteópatas, psiquiatras.

- La compra de productos generales (ropa, comida) y específicos (medicinas, cremas, ortopedia) que precisa la persona dependiente.

- El pago a una tercera persona para cuidados y atención a la persona dependiente y ayuda en tareas del hogar.

- La financiación del coste de servicios sociales utilizados por la persona dependiente (atención domiciliaria, centro de día), así como la adquisición de ayudas técnicas y obras de acondicionamiento del hogar.

- La financiación parcial de la atención y dedicación temporal de la persona familiar cuidadora.

- La financiación de gastos de transporte a distintos recursos sociales y sanitarios, especialmente en el caso de dependientes ubicados en entornos rurales.

- Los gastos generales del hogar (luz, teléfono, comunidad, alimentación).

- El apoyo económico a miembros del hogar en situación de necesidad (por ejemplo, a familiares en paro) que, en distinto grado, colaboran en las tareas de atención.

- Un aporte económico de gran importancia en el caso de hogares en situación precaria o riesgo de precarización.

Atendiendo a los planteamientos que han realizado los agentes de intervención, la PECE ha contribuido a:

- La mejora en la calidad de vida y cuidados de la persona dependiente, ya que permite financiar sus gastos directos (pago de productos específicos, ayudas técnicas), así como los gastos por servicios de terceras personas dedicadas a su cuidado personal o la ayuda en tareas domésticas.
- La mejora de la economía familiar, que en el caso de economías familiares precarias, todavía adquiere un mayor impacto funcional. Se considera que ello redunda en una mejora de la calidad de vida de la familia en general y de la persona dependiente en particular.

- El 'respiro' y la mejora de las condiciones de vida de la persona cuidadora, ya que el uso de la prestación para financiar los servicios de una tercera persona que ayude tanto en los cuidados a la persona dependiente como en las tareas del hogar es un apoyo que permite liberar de ciertas cargas a los familiares cuidadores, concediéndoles la oportunidad de disponer de tiempo propio.

- El reconocimiento social de la labor desarrollada por las personas cuidadoras; es decir, supone que la sociedad reconoce la faceta social del trabajo de cuidado a familiares dependientes, a través de una contraprestación económica y asegurando su cotización a la Seguridad Social. Ello supone una dignificación y puesta en valor de esta función.

- El fomento de la dedicación a los cuidados de la persona dependiente en el caso de familiares con actividad laboral precaria (baja retribución, gran dedicación, escasa flexibilidad horaria), para quienes esta prestación puede operar como estímulo para abandonar esa actividad y centrarse en los cuidados al familiar dependiente.

- El apoyo a la conciliación de labores de cuidado con la actividad laboral, ya que es un recurso que permite costear los servicios de una tercera persona, facilitando la dedicación a la actividad laboral de la persona cuidadora.

Por otro lado, se identifica un discurso que señala la existencia de casos en los que la prestación económica es utilizada para gastos correspondientes a otros miembros del hogar o como complemento general a la economía familiar, pero no está dirigida a sufragar directamente gastos derivados de la atención a la persona dependiente. La máxima desviación respecto al objetivo de la Ley se produciría en aquellos casos en los que la finalidad básica de la prestación es el logro de una fuente de ingresos complementarios, mientras que la persona dependiente no se encuentra debidamente atendida.

Entre los agentes de intervención, también se ha detectado un discurso que atribuye escasa eficiencia social a la PECE, ya que se considera que la atención y los cuidados que recibía la persona dependiente antes de la prestación y los que recibe después son prácticamente iguales (no se registra variación). No obstante, al mismo tiempo, se estima que, salvo excepciones, esa atención es adecuada, es decir, tanto antes como después de la prestación económica la persona dependiente está recibiendo unos cuidados que le permiten mantenerse en su entorno familiar. Otra cosa es que los cuidados que recibe respondan de forma óptima a sus necesidades, o dicho de otra forma, el hecho de que reciba atención en el propio hogar no significa que sea la más adecuada o la que mejor responde a su situación de dependencia. 
En cuanto a la probabilidad de efectos perversos de la PECE entre los miembros de las familias, se ha constatado la posibilidad de fomentar desequilibrios en el reparto de las tareas de cuidados al familiar dependiente, de modo que se perpetúa sobre algún miembro el rol de persona cuidadora, con sus consiguientes problemas y conflictos familiares. La búsqueda de una organización no problemática de las tareas de cuidado entre los miembros de la familia es algo que, en algunos casos, preocupa a los servicios sociales.

Unido a lo anterior, y en referencia a los efectos positivos de esta prestación en las relaciones familiares, se considera interesante, conocer y tener indicadores sobre el grado en que la entrada en vigor de esta ayuda económica ha podido incrementar los niveles de convivencia familiar, con base en la organización de las tareas de cuidado al familiar.

\section{Seguimiento y supervisión de la calidad de la atención y promoción de la autonomía}

\subsection{El espacio supervisor}

En los inicios de la aplicación de la LAPAD, los servicios sociales de base de Gipuzkoa se vieron desbordados de trabajo por el propio 'efecto llamada' de la Ley, constatándose un fuerte incremento de las solicitudes de valoración de dependencia. Dicho 'efecto llamada' ha contribuido a visualizar las situaciones de dependencia existentes en Gipuzkoa y ha permitido generar nuevos clientes para los servicios sociales municipales (con un nuevo perfil ${ }^{12}$ ). Ello ha puesto en evidencia la necesidad de contar con unos mecanismos de control o supervisión respecto a la eficiencia, efectividad o impacto de dichas prestaciones en la calidad de vida de las personas dependientes.

Debemos tener en cuenta que la LAPAD se aprueba y pone en marcha en un contexto de bonanza económica previa a la crisis actual, en el que las administraciones públicas disponían de recursos que favorecían la aplicación inicial de un criterio extensivo de las prestaciones económicas, en el que lo que se priorizaba era la tramitación y reparto de las prestaciones económicas, sin el establecimiento y fijación de un sistema para su seguimiento y supervisión. La demanda de solicitudes y la concesión de prestaciones económicas muy por encima de las previsiones iniciales, en el contexto actual de crisis económica, ha puesto de manifiesto la necesidad de establecer mecanismos de seguimiento y supervisión que evalúen el impacto de estas prestaciones en la

${ }^{12}$ La aplicación de la LAPAD hace que se prodigue el 'etiquetaje’ o 'definición formal' de las personas dependientes. Así, muchas personas dependientes que eran 'atendidas naturalmente' en sus domicilios por sus familiares deciden solicitar la valoración de dependencia con el objeto de acceder, única y exclusivamente, a las prestaciones de económicas (sobre todo las PECE). Este hecho hace que, cada vez en mayor medida, acudan a los servicios sociales de base municipales ‘familias muy normalizadas' con problemáticas de dependencia. mejora de la atención y promoción de la autonomía de las personas dependientes, permitiendo al mismo tiempo adoptar decisiones para la mejora global del sistema de prestaciones sociales a la dependencia.

Desde el punto de vista de la supervisión, es conveniente diferenciar entre lo que es la inspección de carácter administrativo relacionada con el control de los requisitos asociados a la concesión de las prestaciones económicas, y lo que es el seguimiento y la supervisión relacionada con el 'impacto' de esas prestaciones en la calidad de vida de las personas dependientes. En este sentido, lo interesante sería impulsar la función de supervisión vinculada al contacto con las familias, para colaborar en el seguimiento de la situación de dependencia, al objeto de mejorar de forma continua la eficiencia y eficacia del funcionamiento de las prestaciones económicas; es decir, el grado en que están sirviendo para mejorar la calidad de la atención y la autonomía personal de la persona dependiente; o dicho de otro modo, el grado de ajuste entre las necesidades de personas dependientes y el tipo de cuidados y atención que reciben como resultado de estas prestaciones. De esta forma, se pueden detectar y-sobre todo- evitar casos de negligencia o desatención.

\subsection{Factores explicativos del estado de la supervisión}

Desde un punto de vista cualitativo, los principales factores explicativos detectados acerca de la situación y funcionamiento actual de la supervisión de estas prestaciones económica de dependencia en Gipuzkoa son los siguientes:

1. La ausencia de recursos económicos y de personal por parte de los servicios sociales de base municipales que les permitan desarrollar la supervisión o el seguimiento sistemático de las prestaciones económicas y su incidencia en la mejora de la atención y autonomía de las personas dependientes. Esto, a su vez, parece correlacionado con:

- La excesiva carga de trabajo y el desequilibrio entre colectivos y programas gestionados, y los recursos de los que disponen.

- La resistencia a asumir la función de supervisión/control de las prestaciones, por el ‘coste' o ‘desgaste' en imagen y valoración que ello implica para los servicios sociales de base.

2. La propia dificultad intrínseca de supervisar el ámbito privado de los cuidados, así como la carencia de competencia legal y de recursos personales para ello. Unido a esto, también se ha constatado la carencia de un modelo para la supervisión sistemática del impacto específico de las prestaciones económicas, que se manifiesta en:

- La ausencia de una herramienta técnica (protocolo, formulario) que establezca criterios y metodología para desarrollar supervisión o 
seguimiento de las prestaciones económicas, es decir, la fijación de unos parámetros que permitan evaluar la atención y la autonomía de la persona dependiente: tipo de cuidados, intensidad, cualificación/formación de las personas cuidadoras, entre otras cuestiones.

- La insuficiente protección y apoyo normativo a la capacidad de actuación de los servicios sociales de base municipales para poder intervenir directamente en familias con dependientes a su cargo, en supuestos casos en los que se constatan déficits en los cuidados y atención que se prestan a la persona dependiente.

3. La existencia de una supervisión vinculada al seguimiento de las personas usuarias de servicios sociales. Así, el seguimiento y supervisión de las personas perceptoras de prestaciones económicas, en gran medida, se ha centrado en el colectivo de dependientes que también acceden a otro tipo de servicios sociales de base normalizados (servicio de asistencia domiciliaria, centro de día).

4. La falta de seguimiento y supervisión se hace más evidente conforme aumenta el tamaño de los municipios. En este sentido, en los municipios más pequeños el menor número de personas dependientes y el tipo de relaciones existente permiten una mayor individualización y seguimiento de los casos, frente a los municipios mayores, en los que se mezcla un gran número de casos de dependencia con la limitación de recursos de personal para atender la diversidad de programas a los que deben hacer frente.

\subsection{Medidas de control y supervisión detectadas en otros países}

Casi todos los sistemas coinciden en reconocer que una de las principales preocupaciones de las administraciones públicas en cuanto a las fórmulas de asignación económica individual es la dificultad para idear y aplicar medidas orientadas a cuatro objetivos complementarios:

- Garantizar que la atención se presta efectivamente con la intensidad suficiente y que la calidad de la atención así prestada es adecuada.

- Garantizar que las personas cuidadoras ejercen sus funciones en condiciones adecuadas.

- Responder de un uso adecuado de los fondos públicos.

- Responder de la seguridad de las personas.

La preocupación se centra especialmente en los casos en los que las prestaciones económicas son utilizadas para contratar directamente a cuidadores profesionales o para contratar o compensar económicamente a cuidadores informales. En el Cuadro 2, se exponen medidas de control y supervisión que se han detectado en distintos países, clasificadas según momentos, objetivos, aplicabilidad y naturaleza. Ningún sistema aplica todas las medidas; más bien, lo habitual es que sólo integren algunas y que su implementación resulte, todavía, poco sistemática.

\section{Propuestas y recomendaciones}

\subsection{Optimización de la concurrencia de las prestaciones económicas con programas y servicios sociales}

En el ámbito de la mejora y optimización de la concurrencia entre prestaciones económicas y programas y servicios sociales, nos parece importante:

1. Articular una convivencia eficiente y eficaz entre las prestaciones de dependencia y programas y las prestaciones preexistentes en los servicios sociales de Gipuzkoa (Programa Sendian, Programa de Vida Independiente).

2. Garantizar una adecuada estructura de 'copagos' que incentive el uso del recurso que sea más coste-efectivo en cada caso (y estudiar las variaciones de la demanda en función de las aportaciones económicas que deban hacer los colectivos de personas usuarias, teniendo en cuenta sus niveles de renta).

3. Mejorar la coordinación entre recursos sociales y sanitarios (espacio sociosanitario). Ello permitiría prolongar la permanencia de las personas dependientes en su entorno familiar, y ralentizar o disminuir los ingresos en recursos residenciales, con el consiguiente ahorro de costes.

4. Mejorar la información y divulgación del catálogo de servicios sociales, así como la forma y el tipo de servicios que se ofertan, estudiando la viabilidad y el desarrollo futuro de cuestiones como:

- La potenciación de la funcionalidad de servicios sociales como la asistencia domiciliaria o los centros de día, flexibilizando horarios e intensidades de uso.

- La agilización de los procesos de tramitación de ayudas técnicas (grúas, camas articuladas, sillas de ruedas) que haga operativa su solicitud. La demora entre el momento de solicitud y el de entrega disminuye mucho la capacidad de respuesta a necesidades acuciantes.

- Los servicios de asistencia sociosanitaria domiciliaria, como fisioterapeutas, masajistas u osteópatas.

- Los servicios de centros de noche y de comidas a domicilio.

- Los servicios domiciliarios de acompañamiento y salida al exterior: posibilidad de apoyo externo a familias para garantizar el contacto y relación con el medio social (la calle) de la persona dependiente, como forma de prolongar su calidad de vida. 


\begin{tabular}{|c|c|c|c|c|c|c|c|c|c|c|c|c|c|c|c|c|c|c|}
\hline \multirow{3}{*}{ 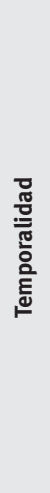 } & \multirow{3}{*}{$\begin{array}{l}\text { Medidas de control y } \\
\text { supervisión }\end{array}$} & \multirow{3}{*}{ Países* } & \multicolumn{8}{|c|}{ Por objetivos } & \multicolumn{4}{|c|}{ Por aplicabilidad } & \multicolumn{4}{|c|}{ Por naturaleza } \\
\hline & & & \multirow{2}{*}{ 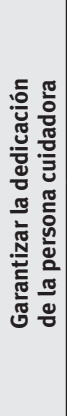 } & \multirow{2}{*}{ 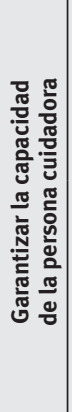 } & \multirow{2}{*}{ 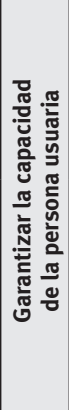 } & \multirow{2}{*}{ 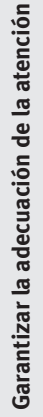 } & \multirow{2}{*}{ 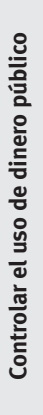 } & \multirow{2}{*}{ 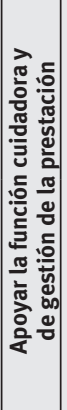 } & \multirow{2}{*}{ 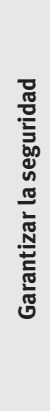 } & \multirow{2}{*}{ 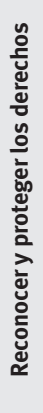 } & \multicolumn{2}{|c|}{$\begin{array}{l}\text { En función } \\
\text { del tipo de } \\
\text { cuidador }\end{array}$} & \multicolumn{2}{|c|}{$\begin{array}{l}\text { En función } \\
\text { del tipo de } \\
\text { prestación }\end{array}$} & \multirow{2}{*}{ 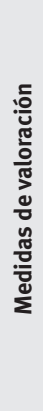 } & \multirow{2}{*}{ 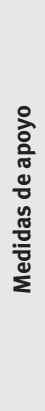 } & \multirow{2}{*}{ 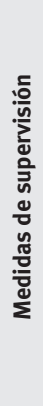 } & \multirow{2}{*}{ 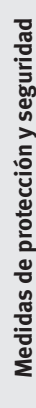 } \\
\hline & & & & & & & & & & & 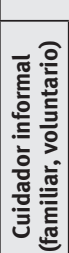 & 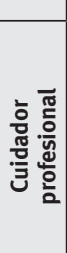 & 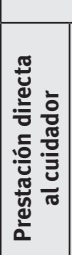 & 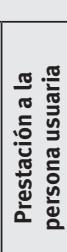 & & & & \\
\hline \multirow{13}{*}{ 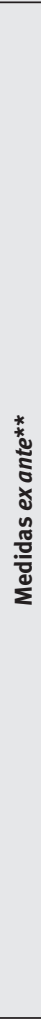 } & $\begin{array}{l}\text { Dedicación de un número } \\
\text { mínimo de horas de } \\
\text { atención }\end{array}$ & $\begin{array}{l}\mathrm{DE}, \mathrm{NL} \\
\mathrm{GB}, \mathrm{SE}\end{array}$ & - & & & & & & & & 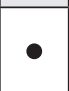 & & - & - & - & & & \\
\hline & $\begin{array}{l}\text { Límites a la dedicación } \\
\text { laboral, formativa o } \\
\text { educativa de la persona } \\
\text { cuidadora } \\
\end{array}$ & $\mathrm{DE}, \mathrm{GB}, \mathrm{SE}$ & $\bullet$ & & & & & & & & 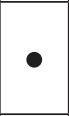 & & - & - & ○ & & & \\
\hline & $\begin{array}{l}\text { Límite al número de } \\
\text { personas atendidas }\end{array}$ & $\mathrm{DE}, \mathrm{GB}, \mathrm{SE}$ & - & & & & & & & & - & & - & & $\bullet$ & & & \\
\hline & Convivencia & $\mathrm{DE}, \mathrm{FR}, \mathrm{NL}$ & 0 & & & & & & & & 0 & & $x$ & 0 & $\bullet$ & & & \\
\hline & $\begin{array}{l}\text { Formalización de la } \\
\text { relación de cuidado }\end{array}$ & \begin{tabular}{|l|} 
FR, NL, SE, \\
US
\end{tabular} & - & & & & & & & $\bullet$ & 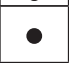 & & - & - & 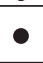 & & $\bullet$ & \\
\hline & $\begin{array}{l}\text { Edad mínima o máxima de } \\
\text { la persona cuidadora }\end{array}$ & NL, GB, SE & & - & & & & & & $\bullet$ & - & & - & - & $\bullet$ & & & - \\
\hline & $\begin{array}{l}\text { Autonomía funcional de la } \\
\text { persona cuidadora }\end{array}$ & NL, US & & - & & & & & & $\bullet$ & $\bullet$ & & $\bullet$ & - & - & & & \\
\hline & $\begin{array}{l}\text { Valoración formal de } \\
\text { la capacidad y de las } \\
\text { necesidades de la persona } \\
\text { cuidadora }\end{array}$ & GB, US & & ○ & & & & & ○ & $\bullet$ & $\bullet$ & & - & $\bullet$ & $\bullet$ & & & $\bullet$ \\
\hline & $\begin{array}{l}\text { Valoración de la } \\
\text { capacidad de gestión y } \\
\text { toma de decisiones de la } \\
\text { persona atendida }\end{array}$ & GB, SE, US & & & $\bullet$ & & & & & & $\bullet$ & $\bullet$ & & $\bullet$ & $\bullet$ & & & $\bullet$ \\
\hline & $\begin{array}{l}\text { Valoración del riesgo de } \\
\text { abuso }\end{array}$ & US & & - & & & & & $\bullet$ & & $\bullet$ & $\bullet$ & $x$ & $\bullet$ & - & & & $\bullet$ \\
\hline & $\begin{array}{l}\text { Previsión de mecanismos } \\
\text { de sustitución del } \\
\text { cuidador }\end{array}$ & GB, US & & & & & & & $\bullet$ & & - & $\bullet$ & $x$ & - & & & & $\bullet$ \\
\hline & $\begin{array}{l}\text { Intervención de los } \\
\text { servicios sociales en el } \\
\text { diseño del paquete de } \\
\text { apoyo }\end{array}$ & $\begin{array}{l}\text { DE, FR, } \\
\text { GB, SE, } \\
\quad \text { US }\end{array}$ & & & & - & $\bullet$ & & & & - & $\bullet$ & & $\bullet$ & $\bullet$ & & $\bullet$ & \\
\hline & $\begin{array}{l}\text { Acreditación y registro de } \\
\text { trabajadores domiciliarios } \\
\text { o asistentes personales }\end{array}$ & FR, US & & - & & & & & $\bullet$ & & & $\bullet$ & & $\bullet$ & 0 & & & $\bullet$ \\
\hline \multirow{8}{*}{ 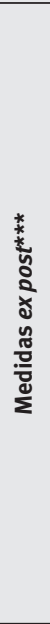 } & $\begin{array}{l}\text { Valoración periódica de } \\
\text { la situación de la persona } \\
\text { dependiente }\end{array}$ & $\begin{array}{l}\text { DE, AT, NL, } \\
\text { US }\end{array}$ & & & & - & & & & & - & - & $x$ & - & - & & - & \\
\hline & $\begin{array}{l}\text { Visitas y entrevistas de } \\
\text { seguimiento }\end{array}$ & \begin{tabular}{|c|}
$\mathrm{DE}, \mathrm{AT}, \mathrm{GB}$ \\
$\mathrm{SE}, \mathrm{US}$
\end{tabular} & & & & - & - & & & & - & $\bullet$ & - & - & & & $\bullet$ & \\
\hline & $\begin{array}{l}\text { Visitas domiciliarias sin } \\
\text { previo aviso }\end{array}$ & US & & & & - & & & - & & - & ○ & $x$ & - & & & $\bullet$ & - \\
\hline & $\begin{array}{l}\text { Control económico del } \\
\text { uso de la prestación } \\
\text { económica }\end{array}$ & $\begin{array}{c}\text { FR, NL, GB, } \\
\text { SE, US }\end{array}$ & & & & & • & & & & - & - & - & $\bullet$ & & & - & \\
\hline & $\begin{array}{l}\text { Servicios de } \\
\text { asesoramiento y apoyo a } \\
\text { la gestión de la prestación } \\
\text { económica }\end{array}$ & $\begin{array}{l}\text { DE, FR, NL, } \\
\text { GB, SE, } \\
\text { US }\end{array}$ & & & & & & - & & & 0 & - & & - & & - & & \\
\hline & $\begin{array}{l}\text { Formación personalizada } \\
\text { domiciliaria para } \\
\text { cuidadores }\end{array}$ & GB, US & & - & & & & - & & & 0 & & ○ & $\bullet$ & & - & & \\
\hline & $\begin{array}{l}\text { Respiro domiciliario para } \\
\text { cuidadores }\end{array}$ & \begin{tabular}{|c|}
$\mathrm{DE}, \mathrm{AT}, \mathrm{FR}$ \\
$\mathrm{NL}, \mathrm{GB}$ \\
$\mathrm{SE}, \mathrm{US}$ \\
\end{tabular} & & • & & & & - & & & - & & - & - & & - & & \\
\hline & $\begin{array}{l}\text { Evaluación global de la } \\
\text { calidad de la atención }\end{array}$ & $\mathrm{NL}, \mathrm{GB}, \mathrm{US}$ & & & & & - & & & & $\bullet$ & 0 & • & 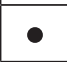 & & & - & \\
\hline
\end{tabular}

* DE: Alemania. AT: Austria. FR: Francia. NL: Países Bajos. GB: Reino Unido. SE: Suecia. US: Estados Unidos.

** Medidas ex ante: las implementadas antes de conceder la prestación y que, por tanto, funcionan como condiciones de acceso.

*** Medidas ex post: las implementadas una vez concedida la prestación económica.

-: supuestos verificados en el marco del análisis comparativo internacional.

X: supuestos que, si bien no se han observado, se consideran aplicables, por analogía.

Fuente: Elaboración propia a partir de SIIS Centro de Documentación y Estudios (2010). 
- Las ayudas específicas para la realización de obras en viviendas y edificios que posibiliten la salida al exterior de la persona dependiente: eliminación de barreras arquitectónicas, obras para inclusión de mobiliario/ equipamiento adecuado.

- La mejora de oferta de recursos dirigidos a personas dependientes con enfermedad mental que responda al déficit existente, impulsando una intervención sociosanitaria de carácter integral.

- Los centros diurnos intermedios con atención específica para personas afectadas por procesos demenciales tipo alzhéimer, que cubran el vacío asistencial existente entre las fases iniciales de la enfermedad y el ingreso en un centro de día.

- Los servicios de apoyo a personas cuidadoras, en la medida en que la calidad de la atención al dependiente depende de las condiciones físicas y mentales de su cuidador/a: programas de formación, grupos de autoayuda, apoyo psicológico, servicios de respiro para fines de semana.

\subsection{Desarrollo y funcionamiento de las prestaciones económicas para la mejora de su impacto}

En cuanto a cuestiones relacionadas con la mejora de los efectos e impactos de las prestaciones económicas de dependencia, se considera fundamental abordar los siguientes aspectos:

1. El establecimiento de mecanismos estandarizados para evaluar el impacto real y sistemático de las prestaciones de las económicas de dependencia, como:

- Elaboración de una herramienta técnica (batería de indicadores): aspectos a valorar y forma de hacerlo.

- Fijación de recursos para abordar la evaluación: personal evaluador.

- Realización de un estudio piloto a una muestra de personas perceptoras.

2. Estudiar la posibilidad de reorientar la PEVS a otros colectivos y destinos: financiación de servicios profesionales de atención domiciliaria (osteopatía, logopedia, homeopatía, podología, masajes).

3. Clarificar el destino de la PECE con el objeto de evitar desviaciones manifiestamente contrarias al objeto de aquélla, que, en el peor de los casos, se asocian a situaciones de desatención de la persona dependiente. Se ha constatado una gran diversidad de destinos a los que se dirige esta prestación (gasto en productos y servicios para la persona dependiente, contraprestación/ compensación a persona cuidadora, contratación de servicios de tercera persona, financiación de servicios sociales, gastos generales del hogar, gastos de otros familiares). Algunos de ellos inciden directamente en la atención a la persona dependiente; otros lo hacen indirectamente; $y$ otros, poco o nada. En este sentido, se plantea:

- Especificar los gastos a los que puede dirigirse la PECE, sobre la base de la mejora en la calidad de vida de la persona dependiente.

- Especificar aquellos gastos o finalidades que no se consideran objeto de la prestación: gastos directos en miembros del hogar sin relación con la persona dependiente (aportación a familiares no cuidadores, gastos del hogar sin repercusiones en la situación de dependencia).

4. Estudiar la posibilidad de ponderar en mayor medida la situación económica del hogar (nivel de ingresos, patrimonio), aproximándose a los criterios aplicados a la hora de acceder a otros servicios sociales.

5. Trabajar el compromiso de asistencia a actividades informativas $\mathrm{y}$ formativas dirigidas a mejorar la capacidad de atención de las personas cuidadoras familiares, mediante el impulso del Programa Sendian y la coordinación con éste. (Ello incidiría, sobre todo, en las familias en las que la percepción de la PECE no implica una modificación en los cuidados y atención que recibe la persona dependiente, es decir, cuando la persona cuidadora sigue prestando la misma atención que prestaba antes de percibir dicha prestación).

6. Trabajar criterios orientados a asegurar una formación y cualificación adecuadas para personas dedicadas a prestar cuidados profesionales a dependientes en el marco de la PEAP, ya que la contratación de una tercera persona a través de la PEAP no implica necesariamente una modificación o mejora en los cuidados y autonomía de la persona dependiente.

7. Procurar un reequilibrio de la expansión de las distintas prestaciones económicas de dependencia (sobre todo, la PECE y la PEAP). Para ello, podría ser interesante:

- Caracterizar contextos de dependencia a los que prioritariamente se debe dirigir cada una de prestaciones (la PECE y la PEAP). Los ejes y variables de caracterización serían:

- Persona dependiente: grado y nivel de dependencia, intensidad (tiempo) mínima precisa para cuidados/atención, grado de especialización de los servicios requeridos.

- Persona cuidadora: situación físicomental (sobre todo de las personas cuidadoras mayores), disponibilidad temporal (dedicación) para cuidados a la persona dependiente, cualificación/ habilidades para el cuidado.

- Contexto social: disponibilidad de red de apoyo familiar y de apoyos sociales.

- Llevar a cabo un desarrollo normativo en consonancia con la caracterización elaborada, 
que permita una orientación de casos por parte de los servicios sociales de base.

\subsection{Mejora de la función de seguimiento y supervisión}

Consideramos que la función de control de las prestaciones económicas debe tener un desarrollo basado en la supervisión entendida como una colaboración positiva entre Administración y familias, de cara a valorar la situación de la persona dependiente y acordar y articular medidas de apoyo que mejoren su atención. En este sentido, debe tenerse en cuenta la ausencia de una cultura de inspección o supervisión en una gran parte de la población perceptora. En consecuencia, a la hora de organizar la función de supervisión se deberían tener en cuenta aspectos como:

- El contacto con las familias por parte de los servicios sociales de base, al objeto de interesarse por la persona dependiente y conocer su evolución y necesidades.

- El trato y la comunicación directa con las familias: visitas, contactos telefónicos.

- El impulso a la comunicación bidireccional: de los servicios sociales a las familias y viceversa.

- En la medida de lo posible, habría que evitar trámites burocráticos y carga administrativa para las familias (documentación, desplazamientos).

En todo caso, consideramos fundamental definir y desarrollar un modelo de supervisión de las prestaciones económicas que permita realizar su segui- miento y evaluación de forma sistemática y homogénea por parte de los distintos servicios sociales de base. Para ello, se plantea abordar los siguientes aspectos:

- Elaboración de una herramienta técnica que establezca criterios y metodología para desarrollar la supervisión: fijación de parámetros que permitan evaluar la calidad de la atención y cuidados prestados:

- Situación de la persona dependiente, cuidados necesarios y destino de la prestación.

- Control de las condiciones administrativas que condicionan la prestación (grado de dependencia, empadronamiento, percepción de otras ayudas).

- Condiciones de la persona cuidadora: capacidad física, formación y cualificación, disposición (temporal y comportamental).

- Intensidad del cuidado: horas dedicadas a la atención.

- Grado de satisfacción de la persona atendida.

- Control de posibles negligencias (que en el ámbito del menor dependiente está muy presente, pero no en el caso de las personas mayores).

- Control de posibles abusos o abandonos (en relación con el fenómeno del maltrato a personas mayores).

- Acuerdo entre distintas instancias para determinar el agente supervisor y la dotación de los recursos económicos y humanos correspondientes.

- Desarrollo normativo para estandarizar, asignar la función supervisora y otorgar autoridad para el desarrollo de esta función. 
(2012): “Real Decreto-Ley 20/2012, de 13 de julio, de medidas para garantizar la estabilidad presupuestaria y de fomento de la competitividad", Boletín Oficial del Estado, nํㅜ 168, 14-7-2012, págs. 50.42850.518 [rhttp://www.boe.es/buscar/doc. php?id=BOE-A-2012-9364'].

(2009) “Decreto Foral 25/2009, de 21 de julio, por el que se regulan las prestaciones económicas de la Ley 39/2006, de 14 de diciembre, de Promoción de la Autonomía Personal y Atención a las personas en situación de dependencia”, Boletín Oficial de Gipuzkoa, no 144, 4-8-2009, pág. 2 [shttp://ssl4. gipuzkoa.net/boletin/asp/ViewRoot.asp?Action $=$ Html\&ltem $=4 \& X=1119181837\rangle]$.

(2007): "Real Decreto 504/2007, de 20 de abril, por el que se aprueba el Baremo de Valoración de la Situación de Dependencia establecido por la Ley 39/2006, de 14 de diciembre, de promoción de la autonomía personal y atención a las personas en situación de dependencia”, Boletín Oficial del Estado, no 96, 21-4-2007, págs. 17.646-17.685 [<http://www.boe.es/ buscar/doc.php?id=BOE-A-2007-8350>].
(2006): “Ley 39/2006, de 14 de diciembre, de Promoción de la Autonomía Personal y Atención a las personas en situación de dependencia", Boletín Oficial del Estado, n- 299, 15-12-2006, págs. 44.142-44.156 [rhttp://www. boe.es/boe/dias/2006/12/15/pdfs/A4414244156.pdf >].

IMSERSO, Sistema para la Autonomía y Atención a la Dependencia. Portal de la Dependencia (s/d): Portal de la Dependencia [«http://www. dependencia.imserso.es/dependencia_01/>].

SIADECO (2012): Estudio cualitativo sobre el impacto de las prestaciones económicas de dependencia en Gipuzkoa, Donostia-San Sebastián, Diputación Foral de Gipuzkoa.

SIIS CENTRO DE DOCUMENTACIÓN Y ESTUDIOS (2010): Prestaciones económicas y otras fórmulas de asignación económica individual en los sistemas de atención a la dependencia. Panorama comparativo internacional, Donostia-San Sebastián, Diputación Foral de Gipuzkoa [/http://www.siis.net/es/ver-detalle. php?ref=192148>]. 